\title{
A HYBRIDIZABLE DISCONTINUOUS GALERKIN METHOD FOR SECOND ORDER ELLIPTIC EQUATIONS WITH DIRAC DELTA SOURCE
}

\author{
Haitao Leng and Yanping Chen*
}

\begin{abstract}
In this paper, we investigate a hybridizable discontinuous Galerkin method for second order elliptic equations with Dirac measures. Under assumption that the domain is convex and the mesh is quasi-uniform, a priori error estimate for the error in $L^{2}$-norm is proved. By duality argument and Oswald interpolation, a posteriori error estimates for the errors in $L^{2}$-norm and $W^{1, p}$-seminorm are also obtained. Finally, numerical examples are provided to validate the theoretical analysis.
\end{abstract}

Mathematics Subject Classification. 49M25, 65K10, 65M50.

Received November 2, 2020. Accepted January 11, 2022.

\section{INTRODUCTION}

In this article, we consider the following problem

$$
\begin{aligned}
-\Delta u & =\delta_{x_{0}} \quad \text { in } \Omega, \\
u & =0 \quad \text { on } \partial \Omega .
\end{aligned}
$$

where $\Omega \subset \mathbb{R}^{d}(d=2,3)$ is an open, bounded, polygonal or polyhedral domain with Lipschitz boundary $\partial \Omega$, and $\delta_{x_{0}}$ is a Dirac measure concentrated at the interior point $x_{0} \in \Omega$. An instance of system (1.1) can be found in the electric field generated by a point charge. Another instance appears in the PDE-constrained optimal control problem [11,12] and in the acoustic monopoles or pollutant transport and degradation in an aquatic media [3].

Since the Dirac function $\delta_{x_{0}}$ does not belong to $H^{-1}(\Omega)$, the solution of problem (1.1) is not in $H^{1}(\Omega)$. In spite of the fact that the solution of problem (1.1) has a very low regularity, it still can be numerically approximated by standard finite element methods. For $d=2$, Babuška [8] obtained the convergence rate $O\left(h^{1-\epsilon}\right)(\epsilon>0)$ for the error in $L^{2}$-norm. In [33], Scott removed $\epsilon$ and yielded the convergence rate $O\left(h^{2-d / 2}\right)$. For an arbitrary Borel measure, Casas derived in [11] a similar result by using different techniques. As for the interior maximum norm error estimates, it has been proved in [34] by Schatz and Wahlbin. It is worth noting that Eriksson [19] proved a priori error estimates for $L^{1}$ - and $W^{1,1}$-errors on adequately refined meshes.

The singular nature of problem (1.1) suggests that the meshes adequately refined around the delta support should be used to boost the accuracy of approximation. In [6], Apel et al. proved the $L^{2}$-error estimates of almost optimal order by using graded meshes on a convex and polygonal domain. In [2], adaptive finite element

Keywords and phrases. Hybridizable discontinuous Galerkin method, a priori error estimate, a posteriori error estimate, elliptic equation, Dirac measure.

School of Mathematical Sciences, South China Normal University, Guangzhou 510631, Guangdong, P.R. China.

*Corresponding author: yanpingchen@scnu.edu.cn 
methods based on a posteriori error estimators were considered for $d=2$. The efficient and reliable a posteriori

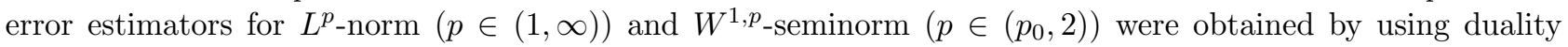
argument, where $p_{0} \in[1,2)$ is a real number depending on the largest inner angle of the domain $\Omega$. For the error in fractional Sobolev space $H^{s}(\Omega)\left(s \in\left(\frac{1}{2}, 1\right)\right)$, the residual type a posteriori error estimators with specifically tailored oscillation were derived by Gaspoz et al. [25]. In [7], Agnelli et al. developed a reliable and efficient a posteriori error estimator for the weighted $H_{\alpha}^{1}$-norm $\left(\alpha \in \mathbb{I} \subset\left(\frac{d}{2}-1, \frac{d}{2}\right)\right)$.

In 2012, Houston and Wihler [26] studied the discontinuous Galerkin (DG) methods for problem (1.1) with $d=2$. The convergence rate $O(h)$ for $L^{2}$-error was proved under a constraint that $x_{0}$ lies in the interior of an element. In addition, a posteriori error estimator, which is efficient and reliable for an extended $L^{2}$-norm, was also shown. It is well known that the DG method is very flexible when it is applied to solve the partial differential equations, however too many globally coupled degrees of freedom are always used and the discrete system is large in particular when the high order polynomial is utilized. Relatively, the HDG methods, proposed by Cockburn et al. [15], not only can keep the advantage of DG methods, but also can get a system with significantly reduced degrees of freedom by introducing the Lagrange multipliers. Currently, it has been used for many problems such as elliptic [13,14], convection diffusion [21], fluid flow [16,28,32], and optimal control $[18,23,29]$, etc.. To the best of our knowledge, there still has no work on error analysis of HDG methods for problems with Dirac measures.

Therefore, in this paper, we investigate error analysis of HDG methods for problem (1.1). In particular, a priori error estimate with convergence rate $O\left(h^{2-d / 2}\right)$ is obtained for the error in $L^{2}$-norm. On the other hand, a posteriori error estimator, which provides an upper and a lower bounds for the error in $L^{2}$-norm, is proved in a convex domain. Moreover, a posteriori error estimator that is efficient and reliable for the error in $W^{1, p}$-seminorm is also derived in a non-convex Lipschitz polygon, where $p \in\left(P^{\Omega}, 2\right)$ and $P^{\Omega}>0$ is a real number depending on the largest inner angle of the domain $\Omega$. Finally, some numerical examples are presented to validate the numerical analysis.

Compared with [2], we need introducing the Oswald interpolation to prove a posteriori error estimator for $W^{1, p}$-seminorm, moreover the a posteriori error estimators obtained in this paper incorporate the term $\left\|u_{h}-\widehat{u}_{h}\right\|_{0, p, \partial K}$ that does not appear in [2], see Lemma 4.4 and Section 4 for more detail, where $\left(u_{h}, \widehat{u}_{h}\right)$ is the HDG solution of problem (1.1). It is worth noting that Oswald interpolation is a very important tool in a posteriori error analysis of HDG methods $[4,5,14,17]$, because it provides a continuous approximation for a discontinuous piecewise polynomial function. Compared with [26], we not only prove a priori and a posteriori error estimates for $L^{2}$-norm in two- and three-dimensional cases, but also derive a posteriori error estimate for $W^{1, p}$-seminorm in two dimensional case.

The rest of this article is arranged as follows: In Section 2, notation and definition corresponding to Sobolev spaces and meshes are provided. In addition, the HDG scheme and some known results are also presented in this section. In Section 3, a priori error estimates are proved. In Sections 4 and 5, the reliability and efficiency of a posteriori error estimators are shown respectively. In Section 6, some numerical examples are presented to validate the numerical analysis. Finally, we end this paper by some conclusions in Section 7.

Throughout this paper, let $c$ with or without subscript be a generic positive constant independent of the mesh size, which may be different in different places. For ease of exposition, we denote $A \leq c B$ by $A \lesssim B$ and $A \approx B$ by $A \lesssim B \lesssim A$.

\section{WEAK FORMULATION AND HDG DISCRETIZATION}

For any bounded and open set $D \subset \mathbb{R}^{d}$ or $D \subset \mathbb{R}^{d-1}, W^{s, q}(D)$ denotes the standard Sobolev space with norm $\|\cdot\|_{s, q, D}$ and seminorm $|\cdot|_{s, q, D}$. When $q=2$, the Sobolev space $W^{s, 2}(D)$ is denoted by $H^{s}(D)$ with norm $\|\cdot\|_{s, D}$ and seminorm $|\cdot|_{s, D}$. If we further have $s=0$, then $H^{0}(D)$ coincides with $L^{2}(D)$, and the inner product is described by $(\cdot, \cdot)_{D}$ for $D \subset \mathbb{R}^{d}$ or $\langle\cdot, \cdot\rangle_{D}$ for $D \subset \mathbb{R}^{d-1}$. For $q \in(1, \infty)$, let $q^{\prime}$ be the conjugate number of $q$ such that $\frac{1}{q}+\frac{1}{q^{\prime}}=1$, then the dual space to $W_{0}^{s, q}(\Omega)$ is denoted by $W^{-s, q^{\prime}}(\Omega)$, where $W_{0}^{s, q}(\Omega)$ is the closure of $C_{0}^{\infty}(\Omega)$ in the space $W^{s, q}(\Omega)$ [1]. If no confusion induced, we also use $\langle\cdot, \cdot\rangle$ to denote the duality pairing between 
spaces $W^{-s, q^{\prime}}(\Omega)$ and $W_{0}^{s, q}(\Omega)$. Finally, we define $H(\operatorname{div}, \Omega):=\left\{\mathbf{v} \in\left(L^{2}(\Omega)\right)^{d}: \nabla \cdot \mathbf{v} \in L^{2}(\Omega)\right\}$, where $\nabla \cdot$ is the divergence operator.

The weak formulation of problem (1.1) is to find $u \in W_{0}^{1, p}(\Omega), p \in\left[1, \frac{d}{d-1}\right)$, such that

$$
a(u, v)=\int_{\Omega} \nabla u \cdot \nabla v d x=v\left(x_{0}\right) \quad \forall v \in W_{0}^{1, p^{\prime}}(\Omega) .
$$

From the embedding theorem, we know that $W_{0}^{1, p^{\prime}}(\Omega) \hookrightarrow \mathcal{C}(\bar{\Omega})$, hence $v\left(x_{0}\right)$ is well-defined.

Remark 2.1. If the domain $\Omega$ is convex, we know from ([11], Thm. 2) and ([22], Thm. 2.1) that the weak formulation (2.1) has a unique weak solution $u \in W_{0}^{1, p}(\Omega)$ with $1 \leq p<\frac{d}{d-1}$. If the domain $\Omega$ is only a polygonal domain with Lipschitz boundary, the aforementioned result is also correct for $d=2$, see ([2], Sect. 2) for more detail.

In order to describe the HDG discretization of problem (2.1), we consider a conforming and shape-regular triangulation $\mathcal{T}_{h}$ of the domain $\Omega$ such that $\bar{\Omega}=\bigcup_{K \in \mathcal{T}_{h}} \bar{K}$. Denote $\mathcal{E}_{h}^{o}$ the set of all interior faces of $\mathcal{T}_{h}$ and $\mathcal{E}_{h}^{\partial}$ the set of all boundary faces. Then we define $\mathcal{E}_{h}=\mathcal{E}_{h}^{o} \cup \mathcal{E}_{h}^{\partial}$ and $\partial \mathcal{T}_{h}=\left\{\partial K: K \in \mathcal{T}_{h}\right\}$, where $\partial K$ denotes the boundary of $K$. For any $K \in \mathcal{T}_{h}$ and $F \in \mathcal{E}_{h}$, let $h_{K}$ and $h_{F}$ be the diameters of $K$ and $F$. Moreover we set $h=\max _{K \in \mathcal{T}_{h}} h_{K}$. For the interior face $F \in \mathcal{E}_{h}^{o}$, we define the jumps $\left.[v]\right|_{F}$ and $\left.[\nabla v \cdot \mathbf{n}]\right|_{F}$ by

$$
\left.[v]\right|_{F}=v^{+}-v^{-},\left.\quad[\nabla v \cdot \mathbf{n}]\right|_{F}=\nabla v^{+} \cdot \mathbf{n}^{+}+\nabla v^{-} \cdot \mathbf{n}^{-}
$$

where $v^{+}$and $v^{-}$are the traces of $v$ on $F=\overline{K^{+}} \cap \overline{K^{-}}$, and $\mathbf{n}^{+}$and $\mathbf{n}^{-}$denote the unit vector normal to the face $F$. As for $F \in \mathcal{E}_{h}^{\partial}$, we formally set $\left.[v]\right|_{F}=\left.v\right|_{F}$ and $\left.[\nabla v \cdot \mathbf{n}]\right|_{F}=\left.(\nabla v)\right|_{F} \cdot \mathbf{n}$. Next, we define the mesh-dependent inner products and norms:

$$
\begin{gathered}
\left(v_{1}, v_{2}\right)_{D}=\sum_{K \in D}\left(v_{1}, v_{2}\right)_{K}, \quad\left\langle\mu_{1}, \mu_{2}\right\rangle_{\partial D}=\sum_{K \in D}\left\langle\mu_{1}, \mu_{2}\right\rangle_{\partial K}, \quad \forall D \subset \mathcal{T}_{h}, \\
\|v\|_{0, p, \mathcal{T}_{h}}=\left(\sum_{K \in \mathcal{T}_{h}}\|v\|_{0, p, K}^{p}\right)^{1 / p}, \quad 1 \leq p<\infty \\
\|(v, \mu)\|_{1, h}^{2}=\|\nabla v\|_{0, \mathcal{T}_{h}}^{2}+\sum_{K \in \mathcal{T}_{h}} h_{K}^{-1}\|v-\mu\|_{L^{2}(\partial K)}^{2},
\end{gathered}
$$

For $k \geq 1$, the discontinuous finite element spaces corresponding to the partition $\mathcal{T}_{h}$ are given as follows:

$$
\begin{aligned}
W_{h}^{k} & =\left\{w \in L^{2}(\Omega):\left.w\right|_{K} \in \mathcal{P}^{k}(K), \forall K \in \mathcal{T}_{h}\right\}, \\
M_{h}^{k} & =\left\{\mu \in L^{2}\left(\mathcal{E}_{h}\right):\left.\mu\right|_{F} \in \mathcal{P}^{k}(F), \forall F \in \mathcal{E}_{h}\right\}, \\
M_{h, 0}^{k} & =\left\{\mu \in M_{h}^{k}:\left.\mu\right|_{F}=0, \forall F \in \mathcal{E}_{h}^{\partial}\right\},
\end{aligned}
$$

where $\mathcal{P}^{k}(D)$ denotes the set of polynomials of degree no larger than $k$ on the domain $D$. Then the HDG discretization of problem (2.1), that approximates the exact solution $\left(u,\left.u\right|_{\mathcal{E}_{h}}\right)$, reads as follows: Find $\left(u_{h}, \widehat{u}_{h}\right) \in$ $W_{h}^{k} \times M_{h, 0}^{k}$ such that

$$
a_{h}\left(u_{h}, \widehat{u}_{h} ; v_{h}, \mu_{h}\right)=\left.\frac{1}{\sharp T_{x_{0}}} \sum_{K \in T_{x_{0}}} v_{h}\right|_{K}\left(x_{0}\right) \quad \forall\left(v_{h}, \mu_{h}\right) \in W_{h}^{k} \times M_{h, 0}^{k},
$$

where $T_{x_{0}}=\left\{K \in \mathcal{T}_{h}: x_{0} \in \bar{K}\right\}, \sharp T_{x_{0}}$ denotes the number of elements in $T_{x_{0}}$, and $a_{h}$ is defined by

$$
\begin{aligned}
a_{h}\left(u_{h}, \widehat{u}_{h} ; v_{h}, \mu_{h}\right)= & \left(\nabla u_{h}, \nabla v_{h}\right)_{\mathcal{T}_{h}}-\left\langle\nabla v_{h} \cdot \mathbf{n}, u_{h}-\widehat{u}_{h}\right\rangle_{\partial \mathcal{T}_{h}} \\
& -\left\langle\nabla u_{h} \cdot \mathbf{n}, v_{h}-\mu_{h}\right\rangle_{\partial \mathcal{T}_{h}}+\left\langle\tau\left(u_{h}-\widehat{u}_{h}\right), v_{h}-\mu_{h}\right\rangle_{\partial \mathcal{T}_{h}} .
\end{aligned}
$$


Here $\tau$ is the stabilization parameter. According to the inverse estimate and trace inequality, we have

$$
\begin{aligned}
a_{h}\left(v_{h}, \mu_{h} ; v_{h}, \mu_{h}\right) & =\left\|\nabla v_{h}\right\|_{0, \Omega}^{2}+\left\|\tau^{1 / 2}\left(v_{h}-\mu_{h}\right)\right\|_{L^{2}\left(\partial \mathcal{T}_{h}\right)}^{2}-2\left\langle\nabla v_{h} \cdot \mathbf{n}, v_{h}-\mu_{h}\right\rangle_{\partial \mathcal{T}_{h}} \\
& \geq \frac{1}{2}\left\|\nabla v_{h}\right\|_{0, \mathcal{T}_{h}}^{2}+\sum_{K \in \mathcal{T}_{h}} \sum_{F \in \partial K}\left(\tau-\frac{c}{h_{K}}\right)\left\|v_{h}-\mu_{h}\right\|_{0, F}^{2},
\end{aligned}
$$

for any $\left(v_{h}, \mu_{h}\right) \in W_{h}^{k} \times M_{h}^{k}$, where $c$ is a constant depending on the polynomial degree and shape-regularity of the mesh. Therefore the bilinear form $a_{h}$ is coercive with respect to the norm $\|\mid(\cdot, \cdot)\| \|_{1, h}$ for $\tau=\frac{\tau_{0}}{h_{F}}$ on each face $F \in \mathcal{E}_{h}$ with $\tau_{0}$ sufficiently large. So the HDG formulation (2.2) has an unique solution $\left(u_{h}, \widehat{u}_{h}\right) \in W_{h}^{k} \times M_{h, 0}^{k}$ for $\tau_{0}$ sufficiently large. Notice that the effect of $\tau_{0}$ on the error will be discussed by numerical experiments in Section 6 .

Now we end this section by introducing some known results that will play an important role in the subsequent proofs.

Lemma 2.2. For each element $K \in \mathcal{T}_{h}$ and any given nonnegative integer $j$, the following estimates hold

$$
\begin{aligned}
& |v|_{i, p, K} \lesssim h_{K}^{-i+\frac{d}{p}-\frac{d}{q}}\|v\|_{q, K}, \quad \forall v \in \mathcal{P}^{j}(K), i=0,1,1 \leq p, q \leq \infty \\
& \|v\|_{0, p, \partial K} \lesssim\|v\|_{0, p, K}^{1-1 / p}\|v\|_{1, p, K}^{1 / p}, \quad \forall v \in W^{1, p}(K), 1 \leq p \leq \infty .
\end{aligned}
$$

Proof. The approximation results (2.4) and (2.5) can be found in Lemma 4.5.3, Theorem 1.6.6 from [9].

Lemma 2.3. Let $I: \mathcal{C}(\bar{\Omega}) \rightarrow V_{h}^{1}$ be the Lagrange interpolation operator, where $V_{h}^{1}$ denotes the space of continuous piecewise linear polynomials. Then we have

$$
\begin{aligned}
& |v-I v|_{i, p, K} \lesssim h_{K}^{2-i}|v|_{2, p, K}, \quad \forall v \in W^{2, p}(K), i=0,1,2, \frac{d}{2}<p<\infty, \\
& |v-I v|_{i, p, K} \lesssim h_{K}^{1-i}|v|_{1, p, K}, \quad \forall v \in W^{1, p}(K), i=0,1, d<p<\infty, \\
& |v-I v|_{0, \infty, K} \lesssim h_{K}^{2-\frac{d}{p}}|v|_{2, p, K}, \quad \forall v \in W^{2, p}(K), \frac{d}{2}<p<\infty, \\
& |v-I v|_{0, \infty, K} \lesssim h_{K}^{1-\frac{d}{p}}|v|_{1, p, K}, \quad \forall v \in W^{1, \infty}(\Omega), d<p<\infty .
\end{aligned}
$$

Proof. The Lagrange interpolation error estimates can be found in Theorem 4.4.4, Corollary 4.4.7 from [9].

Lemma 2.4. Let $\widetilde{I}: W^{s, p} \rightarrow V_{h}^{1}$ be the Scott-Zhang interpolation. Then we have

$$
\sum_{K \in \mathcal{T}_{h}} h_{K}^{p(r-s)}\|v-\widetilde{I} v\|_{r, p, K}^{p} \lesssim|v|_{s, p, \Omega}^{p}, \quad \forall v \in W^{s, p}(\Omega)
$$

where $0 \leq r \leq s \leq 2$ and $1 \leq p \leq \infty$.

Proof. The result can be found in Theorem 4.6.12 from [9].

The next result shows that the polynomial $v_{h} \in W_{h}^{k}$ can be approximated by a continuous function $\widetilde{v}_{h} \in$ $W_{h}^{k} \cap H_{0}^{1}(\Omega)$. It is well-known that this is the so-called Oswald interpolation.

Lemma 2.5. For any $v_{h} \in W_{h}^{k}$, there exists a function $\widetilde{v}_{h} \in W_{h}^{k} \cap H_{0}^{1}(\Omega)$ such that

$$
\begin{gathered}
\sum_{K \in \mathcal{T}_{h}}\left\|v_{h}-\widetilde{v}_{h}\right\|_{0, p, K}^{p} \lesssim \sum_{F \in \mathcal{E}_{h}^{o}} h_{F}\left\|\left[v_{h}\right]\right\|_{0, p, F}^{p}+\sum_{F \in \mathcal{E}_{h}^{\partial}} h_{F}\left\|v_{h}\right\|_{0, p, F}^{p}, \\
\sum_{K \in \mathcal{T}_{h}}\left\|\nabla\left(v_{h}-\widetilde{v}_{h}\right)\right\|_{0, p, K}^{p} \lesssim \sum_{F \in \mathcal{E}_{h}^{o}} h_{F}^{1-p}\left\|\left[v_{h}\right]\right\|_{0, p, F}^{p}+\sum_{F \in \mathcal{E}_{h}^{o}} h_{F}^{1-p}\left\|v_{h}\right\|_{0, p, F}^{p},
\end{gathered}
$$

for $1 \leq p<\infty$. 
Proof. Here we only prove the error estimate (2.12). As for the error estimate (2.11), it can be proved similarly.

For the case of $p=2$, the approximation (2.12) has been proved in Theorem 2.2 from [27]. As for other cases, the method of proof is similar.

Let $\mathcal{N}_{K}=\left\{x_{K}^{(j)}, j=1, \cdots, m\right\}$ be the Lagrange nodes of $K$ and $\left\{\phi_{K}^{(j)}, j=1, \cdots, m\right\}$ the corresponding Lagrange basis functions. Then we have $v_{h}=\sum_{K \in \mathcal{T}_{h}} \sum_{j=1}^{m} \alpha_{K}^{(j)} \phi_{K}^{(j)}$ and $\widetilde{v}_{h}=\sum_{\nu \in \mathcal{N}} \beta^{(\nu)} \phi^{(\nu)}$, where $\mathcal{N}=$ $\bigcup_{K \in \mathcal{T}_{h}} \mathcal{N}_{K}$ and $\phi^{(\nu)}$ is the Lagrange basis function of the node $\nu$. Here $\beta^{(\nu)}$ is defined as that in Theorem 2.2 from [27]. Hence

$$
\begin{aligned}
\sum_{K \in \mathcal{T}_{h}}\left\|\nabla\left(v_{h}-\widetilde{v}_{h}\right)\right\|_{0, p, K}^{p} & =\sum_{K \in \mathcal{T}_{h}} \int_{K}\left|\sum_{j=1}^{m}\left(\alpha_{K}^{(j)}-\beta_{K}^{(j)}\right) \nabla \phi_{K}^{(j)}\right|^{p} \mathrm{dx} \\
& \lesssim \sum_{K \in \mathcal{T}_{h}} \sum_{j=1}^{m}\left|\alpha_{K}^{(j)}-\beta_{K}^{(j)}\right|^{p}\left\|\nabla \phi_{K}^{(j)}\right\|_{0, p, K}^{p},
\end{aligned}
$$

where $\beta_{K}^{(j)}=\beta^{(\nu)}$ whenever $x_{K}^{(j)}=\nu$. Since $\left\|\nabla \phi_{K}^{(j)}\right\|_{0, p, K} \lesssim h_{K}^{\frac{d}{p}-1}$, the above inequality and inverse estimate (2.4) yield

$$
\begin{aligned}
\sum_{K \in \mathcal{T}_{h}}\left\|\nabla\left(v_{h}-\widetilde{v}_{h}\right)\right\|_{0, p, K}^{p} & \lesssim \sum_{K \in \mathcal{T}_{h}} h_{K}^{d-p} \sum_{j=1}^{m}\left|\alpha_{K}^{(j)}-\beta_{K}^{(j)}\right|^{p} \\
& \lesssim \sum_{F \in \mathcal{E}_{h}^{o}} h_{F}^{d-p}\left\|\left[v_{h}\right]\right\|_{0, \infty, F}^{p}+\sum_{F \in \mathcal{E}_{h}^{\partial}} h_{F}^{d-p}\left\|v_{h}\right\|_{0, \infty, F}^{p} \\
& \lesssim \sum_{F \in \mathcal{E}_{h}^{o}} h_{F}^{1-p}\left\|\left[v_{h}\right]\right\|_{0, p, F}^{p}+\sum_{F \in \mathcal{E}_{h}^{\partial}} h_{F}^{1-p}\left\|v_{h}\right\|_{0, p, F}^{p}
\end{aligned}
$$

\section{A PRIORI ERROR ANALYSIS}

This section mainly focuses on a priori error analysis for the error $\left\|u-u_{h}\right\|_{0, \Omega}$. To this end, we introduce the following auxiliary problem:

$$
\begin{aligned}
-\Delta \phi_{f}=f & \text { in } \Omega, \\
\phi_{f}=0 & \text { on } \partial \Omega .
\end{aligned}
$$

Theorem 3.1. The problem (3.1) has an unique weak solution which holds the following regularities from the different cases:

(i) If $f \in L^{2}(\Omega)$ and the domain $\Omega$ is convex, we have

$$
\phi_{f} \in H^{2}(\Omega) \cap H_{0}^{1}(\Omega) \quad \text { and } \quad\left\|\phi_{f}\right\|_{2, \Omega} \lesssim\|f\|_{0, \Omega} .
$$

(ii) If $f \in L^{p^{\prime}}(\Omega), d=2$ and the domain $\Omega$ is a non-convex Lipschtiz polygon, we have

$$
\phi_{f} \in W_{0}^{2, p^{\prime}}(\Omega) \quad \text { and } \quad\left|\phi_{f}\right|_{2, p^{\prime}, \Omega} \lesssim\|f\|_{0, p^{\prime}, \Omega},
$$

where $\left(2-\frac{\pi}{\theta}\right) p^{\prime}<2$ and $\theta>\pi$ is the largest inner angle of the domain $\Omega$.

Proof. The result of case (i) is well-known [10]. The result of case (ii) can be found in Section 4 from [2] and Theorem 4.4.4.13 from [24]. 
Following the approach in [26,33], we define $\delta_{h} \in W_{h}^{k}$ such that $\delta_{h}=0$ on $\mathcal{T}_{h} \backslash T_{x_{0}}$, and

$$
\int_{K} \delta_{h} v_{h} d x=\left.v_{h}\right|_{K}\left(x_{0}\right) \quad \forall v_{h} \in \mathcal{P}^{k}(K), K \in T_{x_{0}} .
$$

Then with ([26], Subsect. 3.1) and inverse estimate (2.4), we can obtain

$$
\left\|\delta_{h}\right\|_{0, K} \approx h_{K}^{-d / 2}, \quad \forall K \in T_{x_{0}} .
$$

Furthermore, we define $\phi_{\delta_{h}}$ as the solution of problem (3.1) with $f=\frac{1}{\sharp T_{x_{0}}} \delta_{h}$, and the weak formulation is to find $\phi_{\delta_{h}} \in H_{0}^{1}(\Omega)$ such that

$$
a\left(\phi_{\delta_{h}}, v\right)=\frac{1}{\sharp T_{x_{0}}} \int_{\Omega} \delta_{h} v \mathrm{dx}, \quad \forall v \in H_{0}^{1}(\Omega) .
$$

From now on, we assume in the rest of this section that the domain $\Omega$ is convex and the partition $\mathcal{T}_{h}$ is quasi-uniform.

By a simple calculation, we can find $u-u_{h}=u-\phi_{\delta_{h}}+\phi_{\delta_{h}}-u_{h}$, hence a priori error analysis for the error $\left\|u-u_{h}\right\|_{0, \Omega}$ can be divided into two parts. Firstly, we are going to deal with the error $\left\|u-\phi_{\delta_{h}}\right\|_{0, \Omega}$.

Lemma 3.2. Let $u \in W_{0}^{1, p}(\Omega)\left(p \in\left[1, \frac{d}{d-1}\right)\right)$ and $\phi_{\delta_{h}} \in H_{0}^{1}(\Omega)$ be the solutions of problems (2.1) and (3.6). If the domain $\Omega$ is convex and the mesh is quasi-uniform, it holds that

$$
\left\|u-\phi_{\delta_{h}}\right\|_{0, \Omega} \lesssim h^{2-d / 2} .
$$

Proof. Let $\phi_{f}$ be the solution of problem (3.1) with $f \in L^{2}(\Omega)$. Let $\phi_{f, h} \in V_{h}^{1}$ and $\phi_{\delta_{h}, h} \in V_{h}^{1}$ be the standard finite element approximations of $\phi_{f}$ and $\phi_{\delta_{h}}$. Then we have the following standard error estimates (see, e.g., $[10])$ :

$$
\begin{aligned}
& \left\|\phi_{f}-\phi_{f, h}\right\|_{L^{\infty}(\Omega)} \lesssim h^{2-d / 2}\|f\|_{0, \Omega}, \\
& \left\|\phi_{f}-\phi_{f, h}\right\|_{0, \Omega}+h\left\|\phi_{f}-\phi_{f, h}\right\|_{1, \Omega} \lesssim h^{2}\left\|\phi_{f}\right\|_{2, \Omega} \lesssim h^{2}\|f\|_{0, \Omega} .
\end{aligned}
$$

Moreover, integration by parts can yield

$$
\begin{aligned}
\int_{\Omega}\left(u-\phi_{\delta_{h}}\right) f & =\int_{\Omega} \nabla\left(u-\phi_{\delta_{h}}\right) \cdot \nabla\left(\phi_{f}-\phi_{f, h}\right)+\int_{\Omega} \nabla\left(u-\phi_{\delta_{h}}\right) \cdot \nabla \phi_{f, h} \\
& =\int_{\Omega} \nabla\left(u-\phi_{\delta_{h}}\right) \cdot \nabla\left(\phi_{f}-\phi_{f, h}\right) \\
& =\phi_{f}\left(x_{0}\right)-\phi_{f, h}\left(x_{0}\right)+\int_{\Omega} \nabla\left(\phi_{\delta_{h}, h}-\phi_{\delta_{h}}\right) \cdot \nabla\left(\phi_{f}-\phi_{f, h}\right) \\
& \lesssim h^{2-d / 2}\|f\|_{0, \Omega}+h^{2-d / 2}\|f\|_{0, \Omega},
\end{aligned}
$$

by (3.5), (3.7) and (3.8), which concludes the proof.

Now the remaining task is to estimate the error $\left\|\phi_{\delta_{h}}-u_{h}\right\|_{0, \Omega}$. Obviously, according to the definitions of $\phi_{\delta_{h}}$ and $\delta_{h}$, we know that $\left(u_{h}, \widehat{u}_{h}\right)$ is an HDG approximation of $\phi_{\delta_{h}}$. Hence from ([30], Thm. 2), we have

$$
\left\|\phi_{\delta_{h}}-u_{h}\right\|_{0, \Omega} \lesssim h^{2}\left|\phi_{\delta_{h}}\right|_{2, \Omega},
$$

which, together with (3.5) and Lemma 3.2, yields the following a priori error estimate.

Theorem 3.3. Let $u$ and $\left(u_{h}, \widehat{u}_{h}\right)$ be the solutions of problems (2.1) and (2.2). If the domain $\Omega$ is convex and the mesh is quasi-uniform, we have

$$
\left\|u-u_{h}\right\|_{0, \Omega} \lesssim h^{2-d / 2}
$$




\section{Reliability of a POSTERIORI ERROR ESTIMATORS}

In this section, we consider a posteriori error analysis for the HDG scheme (2.2). Specifically, we will prove a posteriori error estimators for the errors $\left\|u-u_{h}\right\|_{0, \Omega}$ and $\left\|\nabla\left(u-u_{h}\right)\right\|_{s, \Omega}$.

If $x_{0}$ is a node of the partition $\mathcal{T}_{h}$, we define the local error estimators $\eta_{K}$ and $\zeta_{K, s}$ by

$$
\begin{aligned}
& \eta_{K}^{2}=h_{K}^{4}\left\|\Delta u_{h}\right\|_{0, K}^{2}+\tau_{0}^{2} h_{K}\left\|u_{h}-\widehat{u}_{h}\right\|_{0, \partial K}^{2}, \\
& \zeta_{K, s}^{s}=h_{K}^{s}\left\|\Delta u_{h}\right\|_{0, s, K}^{s}+\tau_{0}^{s} h_{K}^{1-s}\left\|u_{h}-\widehat{u}_{h}\right\|_{0, s, \partial K}^{s},
\end{aligned}
$$

otherwise,

$$
\begin{gathered}
\eta_{K}^{2}= \begin{cases}h_{K}^{4-d}+h_{K}^{4}\left\|\Delta u_{h}\right\|_{0, K}^{2}+\tau_{0}^{2} h_{K}\left\|u_{h}-\widehat{u}_{h}\right\|_{0, \partial K}^{2} & \text { if } x_{0} \in \bar{K}, \\
h_{K}^{4}\left\|\Delta u_{h}\right\|_{0, K}^{2}+\tau_{0}^{2} h_{K}\left\|u_{h}-\widehat{u}_{h}\right\|_{0, \partial K}^{2} & \text { otherwise, }\end{cases} \\
\zeta_{K, s}^{s}= \begin{cases}h_{K}^{2-s}+h_{K}^{s}\left\|\Delta u_{h}\right\|_{0, s, K}^{s}+\tau_{0}^{s} h_{K}^{1-s}\left\|u_{h}-\widehat{u}_{h}\right\|_{0, s, \partial K}^{s} & \text { if } x_{0} \in \bar{K}, \\
h_{K}^{s}\left\|\Delta u_{h}\right\|_{0, s, K}^{s}+\tau_{0}^{s} h_{K}^{1-s}\left\|u_{h}-\widehat{u}_{h}\right\|_{0, s, \partial K}^{s} & \text { otherwise. }\end{cases}
\end{gathered}
$$

Before proving a posteriori error estimates, we first show the following properties for the HDG scheme (2.2).

Lemma 4.1. Let $\left(u_{h}, \widehat{u}_{h}\right)$ be the solution of problem (2.2), then we have

$$
\begin{aligned}
{\left.\left[\nabla u_{h} \cdot \boldsymbol{n}\right]\right|_{F}=} & \frac{\tau_{0}}{h_{F}}\left(u_{h}^{+}-\widehat{u}_{h}\right)+\frac{\tau_{0}}{h_{F}}\left(u_{h}^{-}-\widehat{u}_{h}\right), \quad \mathcal{E}_{h}^{o} \ni F=\overline{K^{+}} \cap \overline{K^{-}}, \\
\left(\Delta u_{h}, v_{h}\right)_{\mathcal{T}_{h}}= & -\left\langle\nabla v_{h} \cdot \boldsymbol{n}, u_{h}-\widehat{u}_{h}\right\rangle_{\partial \mathcal{T}_{h}}+\sum_{K \in \mathcal{T}_{h}} \sum_{F \in \partial K} \frac{\tau_{0}}{h_{F}}\left\langle u_{h}-\widehat{u}_{h}, v_{h}\right\rangle_{F} \\
& -\left.\frac{1}{\sharp T_{x_{0}}} \sum_{K \in T_{x_{0}}} v_{h}\right|_{K}\left(x_{0}\right),
\end{aligned}
$$

for any $v_{h} \in W_{h}^{k}$.

Proof. By setting $v_{h}=0$ in $(2.2)$, we arrive at

$$
\left\langle\nabla u_{h} \cdot \mathbf{n}, \mu_{h}\right\rangle_{\partial \mathcal{T}_{h}}-\left\langle\tau\left(u_{h}-\widehat{u}_{h}\right), \mu_{h}\right\rangle_{\partial \mathcal{T}_{h}}=0, \quad \forall \mu_{h} \in M_{h, 0}^{k},
$$

where $\tau=\frac{\tau_{0}}{h_{F}}$ on each face $F \in \mathcal{E}_{h}$. Hence the result (4.1) can be obtained by the above equality. Moreover the equality (4.2) can be derived directly by using (4.1), (2.2) and integration by parts.

Now we are going to prove a posteriori error estimate for the error $\left\|u-u_{h}\right\|_{0, \Omega}$ by the duality argument.

Lemma 4.2. Let $u$ and $\left(u_{h}, \widehat{u}_{h}\right)$ be the solutions of problems (2.1) and (2.2). If the domain $\Omega$ is convex, we have

$$
\left\|u-u_{h}\right\|_{0, \Omega} \lesssim\left(\sum_{K \in \mathcal{T}_{h}} \eta_{K}^{2}\right)^{1 / 2} .
$$

Proof. Let $\phi_{f}$ be the solution of problem (3.1) with $f \in L^{2}(\Omega)$. From the case (i) of Theorem 3.1, we know that the solution $\phi_{f}$ satisfies the regularity (3.2). Hence by integration by parts, we arrive at

$$
\begin{aligned}
\left(u-u_{h}, f\right)_{\Omega} & =\left(\nabla u, \nabla \phi_{f}\right)_{\Omega}+\left(u_{h}, \Delta \phi_{f}\right)_{\mathcal{T}_{h}} \\
& =\phi_{f}\left(x_{0}\right)+\left(\Delta u_{h}, \phi_{f}\right)_{\mathcal{T}_{h}}+\left\langle\nabla \phi_{f} \cdot \mathbf{n}, u_{h}\right\rangle_{\partial \mathcal{T}_{h}}-\left\langle\nabla u_{h} \cdot \mathbf{n}, \phi_{f}\right\rangle_{\partial \mathcal{T}_{h}} .
\end{aligned}
$$


Insert (4.2) in the above equality to yield

$$
\begin{aligned}
\left(u-u_{h}, f\right)_{\Omega}= & \phi_{f}\left(x_{0}\right)-\left.\frac{1}{\sharp T_{x_{0}}} \sum_{K \in T_{x_{0}}} v_{h}\right|_{K}\left(x_{0}\right)+\left(\Delta u_{h}, \phi_{f}-v_{h}\right)_{\mathcal{T}_{h}} \\
& +\left\langle\nabla \phi_{f} \cdot \mathbf{n}, u_{h}\right\rangle_{\partial \mathcal{T}_{h}}-\left\langle\nabla v_{h} \cdot \mathbf{n}, u_{h}-\widehat{u}_{h}\right\rangle_{\partial \mathcal{T}_{h}} \\
& -\left\langle\nabla u_{h} \cdot \mathbf{n}, \phi_{f}\right\rangle_{\partial \mathcal{T}_{h}}+\left\langle\tau\left(u_{h}-\widehat{u}_{h}\right), v_{h}\right\rangle_{\partial \mathcal{T}_{h}} \\
= & \left(\phi_{f}\left(x_{0}\right)-\left.\frac{1}{\sharp T_{x_{0}}} \sum_{K \in T_{x_{0}}} v_{h}\right|_{K}\left(x_{0}\right)\right)+\left(\Delta u_{h}, \phi_{f}-v_{h}\right)_{\mathcal{T}_{h}} \\
& +\left\langle\nabla\left(\phi_{f}-v_{h}\right) \cdot \mathbf{n}, u_{h}-\widehat{u}_{h}\right\rangle_{\partial \mathcal{T}_{h}}+\left\langle\tau\left(u_{h}-\widehat{u}_{h}\right), v_{h}-\phi_{f}\right\rangle_{\partial \mathcal{T}_{h}} \\
= & I_{1}+I_{2}+I_{3}+I_{4},
\end{aligned}
$$

for any $v_{h} \in W_{h}^{k}$. Note that we have used (4.1) and the fact that $\nabla \phi_{f} \in H(\operatorname{div}, \Omega)$ in the derivation of (4.4). Let $v_{h}=I \phi_{f}$ in (4.4), we can get by (2.5) and (2.6)

$$
I_{2} \lesssim \sum_{K \in \mathcal{T}_{h}} h_{K}^{2}\left\|\Delta u_{h}\right\|_{0, K}\left|\phi_{f}\right|_{2, K}
$$

and

$$
\begin{aligned}
I_{3}+I_{4} \lesssim & \sum_{K \in \mathcal{T}_{h}}\left(\left\|\nabla\left(\phi_{f}-I \phi_{f}\right)\right\|_{0, K}^{1 / 2}\left\|\nabla\left(\phi_{f}-I \phi_{f}\right)\right\|_{1, K}^{1 / 2}\right)\left\|u_{h}-\widehat{u}_{h}\right\|_{0, \partial K} \\
& +\sum_{K \in \mathcal{T}_{h}} \frac{\tau_{0}}{h_{K}}\left(\left\|\phi_{f}-I \phi_{f}\right\|_{0, K}^{1 / 2}\left\|\phi_{f}-I \phi_{f}\right\|_{1, K}^{1 / 2}\right)\left\|u_{h}-\widehat{u}_{h}\right\|_{0, \partial K} \\
\lesssim & \sum_{K \in \mathcal{T}_{h}} \tau_{0} h_{K}^{1 / 2}\left\|u_{h}-\widehat{u}_{h}\right\|_{0, \partial K}\left|\phi_{f}\right|_{2, K} .
\end{aligned}
$$

As for the term $I_{1}$, if $x_{0}$ is a node of the partition $\mathcal{T}_{h}$, we have $I_{1}=0$, otherwise, the Lagrange interpolation error estimate (2.8) can get

$$
I_{1}=\frac{1}{\sharp T_{x_{0}}} \sum_{K \in T_{x_{0}}}\left(\phi_{f}\left(x_{0}\right)-\left.I \phi_{f}\right|_{K}\left(x_{0}\right)\right) \leq \frac{1}{\sharp T_{x_{0}}} \sum_{K \in T_{x_{0}}}\left\|\phi_{f}-I \phi_{f}\right\|_{0, \infty, K} \lesssim \frac{1}{\sharp T_{x_{0}}} \sum_{K \in T_{x_{0}}} h_{K}^{2-\frac{d}{2}}\left|\phi_{f}\right|_{2, K} .
$$

Hence the result (4.3) can be achieved by using (3.2) and (4.4)-(4.7).

Remark 4.3. Since the function $\nabla \phi_{f}$ does not belong to $H(d i v, \Omega)$ for any $\phi_{f} \in W_{0}^{2, p^{\prime}}(\Omega)\left(p^{\prime}<2\right)$, the additional term $\left\langle\nabla \phi_{f} \cdot \mathbf{n}, \widehat{u}_{h}\right\rangle_{\partial \mathcal{T}_{h}}$ can not be eliminated when a posteriori error estimate of $\left\|u-u_{h}\right\|_{0, p, \Omega}\left(P_{\Omega}<\right.$ $p<\infty$ ) is proved by using the result introduced in case (ii) of Theorem 3.1, where $P_{\Omega}=\frac{2 \theta}{\pi}>2$. This is why we have not provided a posteriori error analysis for $\left\|u-u_{h}\right\|_{0, p, \Omega}\left(P_{\Omega}<p<\infty\right)$.

In order to prove a posteriori error estimate for $\left\|\nabla\left(u-u_{h}\right)\right\|_{0, p, \Omega}$ in two-dimensional case, we consider a problem that is the same with that in [2]: Find $w \in W_{0}^{1, p^{\prime}}(\Omega)$ such that

$$
a(w, v)=\int_{\Omega} \Psi \cdot \nabla v, \quad \forall v \in W_{0}^{1, p}(\Omega)
$$

for $\Psi \in\left(L^{p^{\prime}}(\Omega)\right)^{2}$. From [2], we know that if $d=2$ and the domain $\Omega$ is a Lipschtiz polygon, the problem (4.8) has an unique weak solution $w \in W_{0}^{1, p^{\prime}}(\Omega)$ satisfying

$$
|w|_{1, p^{\prime}, \Omega} \lesssim\|\Psi\|_{0, p^{\prime}, \Omega}
$$

for $p \in\left(P^{\Omega}, 2\right)$, where $P^{\Omega}:=\max \left\{1,2 /\left(1+\frac{\pi}{\theta}\right)\right\}, \theta$ is the largest inner angle of the domain $\Omega$ and $\frac{1}{p}+\frac{1}{p^{\prime}}=1$. 
Lemma 4.4. Let $u$ and $\left(u_{h}, \widehat{u}_{h}\right)$ be the solutions of problems (2.1) and (2.2). If $d=2$ and the domain $\Omega$ is a Lipschitz polygon, the following error estimate holds

$$
\left\|\nabla\left(u-u_{h}\right)\right\|_{0, p, \mathcal{T}_{h}} \lesssim\left(\sum_{K \in \mathcal{T}_{h}} \zeta_{K, p}^{p}\right)^{1 / p}
$$

where $p \in\left(P^{\Omega}, 2\right)$.

Proof. Since $u_{h}$ does not belong to the space $W^{1, p}(\Omega)$, we can not apply the duality argument directly for $u-u_{h}$ by (4.8). Here, let $\widetilde{u}_{h}$ be the Oswald interpolation of $u_{h}$, then using duality argument for $u-\widetilde{u}_{h}$ we have

$$
\begin{aligned}
\int_{\Omega} \nabla\left(u-\widetilde{u}_{h}\right) \cdot \Psi \mathrm{dx} & =\left(\nabla\left(u-\widetilde{u}_{h}\right), \nabla w\right)_{\Omega} \\
& =w\left(x_{0}\right)+\left(\nabla\left(u_{h}-\widetilde{u}_{h}\right), \nabla w\right)_{\mathcal{T}_{h}}-\left(\nabla u_{h}, \nabla w\right)_{\mathcal{T}_{h}} \\
& =w\left(x_{0}\right)+\left(\nabla\left(u_{h}-\widetilde{u}_{h}\right), \nabla w\right)_{\mathcal{T}_{h}}+\left(\Delta u_{h}, w\right)_{\mathcal{T}_{h}}-\left\langle\nabla u_{h} \cdot \mathbf{n}, w\right\rangle_{\partial \mathcal{T}_{h}}
\end{aligned}
$$

Insert (4.2) in the above equality to infer that

$$
\begin{aligned}
\int_{\Omega} \nabla\left(u-\widetilde{u}_{h}\right) \cdot \Psi \mathrm{dx}= & \left(w\left(x_{0}\right)-\left.\frac{1}{\sharp T_{x_{0}}} \sum_{K \in T_{x_{0}}} v_{h}\right|_{K}\left(x_{0}\right)\right)+\left(\Delta u_{h}, w-v_{h}\right)_{\mathcal{T}_{h}} \\
& +\left(\nabla\left(u_{h}-\widetilde{u}_{h}\right), \nabla w\right)_{\mathcal{T}_{h}}-\left\langle\nabla v_{h} \cdot \mathbf{n}, u_{h}-\widehat{u}_{h}\right\rangle_{\partial \mathcal{T}_{h}} \\
& +\left\langle\tau\left(u_{h}-\widehat{u}_{h}\right), v_{h}-w\right\rangle_{\partial \mathcal{T}_{h}} \\
= & E_{1}+E_{2}+E_{3}+E_{4}+E_{5} .
\end{aligned}
$$

for any $v_{h} \in W_{h}^{k}$.

Let $v_{h}=I w \in V_{h}^{1}$ in (4.10), the trace inequality (2.5) and Lagrange interpolation error estimate (2.7) can get

$$
E_{2} \lesssim \sum_{K \in \mathcal{T}_{h}} h_{K}\left\|\Delta u_{h}\right\|_{0, p, K}|w|_{1, p^{\prime}, K}
$$

and

$$
\begin{aligned}
E_{4}+E_{5} \lesssim & \sum_{K \in \mathcal{T}_{h}}\left\|u_{h}-\widehat{u}_{h}\right\|_{0, p, \partial K}\left(\|\nabla I w\|_{0, p^{\prime}, K}^{\frac{1}{p}}\|\nabla I w\|_{1, p^{\prime}, K}^{\frac{1}{p^{\prime}}}\right. \\
& \left.+\frac{\tau_{0}}{h_{K}}\|w-I w\|_{0, p^{\prime}, K}^{\frac{1}{p}}\|w-I w\|_{1, p^{\prime}, K}^{\frac{1}{p^{\prime}}}\right) \\
\lesssim & \sum_{K \in \mathcal{T}_{h}} \tau_{0} h_{K}^{\frac{1}{p}-1}\left\|u_{h}-\widehat{u}_{h}\right\|_{0, p, \partial K}|w|_{1, p^{\prime}, K} .
\end{aligned}
$$

If $x_{0}$ is a node of the partition $\mathcal{T}_{h}, E_{1}=0$, otherwise, we have

$$
E_{1} \leq \frac{1}{\sharp T_{x_{0}}} \sum_{K \in T_{x_{0}}}\|w-I w\|_{0, \infty, K} \lesssim \sum_{K \in T_{x_{0}}} h_{K}^{\frac{2}{p}-1}|w|_{1, p^{\prime}, K} .
$$


by the Lagrange interpolation error estimate (2.9). As for the term $E_{3}$, the Oswald interpolation error estimate (2.12) can yield

$$
\begin{aligned}
E_{3} & \lesssim\left(\sum_{K \in \mathcal{T}_{h}}\left\|\nabla\left(u_{h}-\widetilde{u}_{h}\right)\right\|_{0, p, K}^{p}\right)^{1 / p}|w|_{1, p^{\prime}, \Omega} \\
& \lesssim\left(\sum_{F \in \mathcal{E}_{h}^{o}} h_{F}^{1-p}\left\|\left[u_{h}\right]\right\|_{0, p, F}^{p}+\sum_{F \in \mathcal{E}_{h}^{\partial}} h_{F}^{1-p}\left\|u_{h}\right\|_{0, p, F}^{p}\right)^{1 / p}|w|_{1, p^{\prime}, \Omega} \\
& \lesssim\left(\sum_{K \in \mathcal{T}_{h}} h_{K}^{1-p}\left\|u_{h}-\widehat{u}_{h}\right\|_{0, p, \partial K}^{p}\right)^{1 / p}|w|_{1, p^{\prime}, \Omega} .
\end{aligned}
$$

Hence combining (4.9) and (4.10)-(4.14), we have

$$
\left\|\nabla\left(u-\widetilde{u}_{h}\right)\right\|_{0, p, \Omega} \lesssim\left(\sum_{K \in \mathcal{T}_{h}} \zeta_{K, p}^{p}\right)^{1 / p},
$$

which, together with the Oswald interpolation error estimate (2.12) and the triangle inequality, concludes the proof.

\section{EFFICIENCY OF A POSTERIORI ERROR ESTIMATORS}

In this section, we mainly prove the efficiency of a posteriori error estimators. Before this, we first introduce the element and face bubble functions $B_{K}$ and $B_{F}$ as that in [35]. Following the approach in [2,20], we define

$$
\psi_{K}(x)= \begin{cases}B_{K}^{2}(x) \frac{\left|x-x_{0}\right|^{2}}{h_{K}^{2}} & \text { if } x_{0} \in K, \\ B_{K}^{2}(x) & \text { otherwise }\end{cases}
$$

for any $K \in \mathcal{T}_{h}$, and

$$
\psi_{F}(x)= \begin{cases}B_{F}^{2}(x) \frac{\left|x-x_{0}\right|^{2}}{h_{F}^{2}} & \text { if } x_{0} \in w_{F}, \\ B_{F}^{2}(x) & \text { otherwise }\end{cases}
$$

for $F \in \mathcal{E}_{h}^{o}$, where $w_{F}:=\bigcup\left\{K: F \subset \partial K, K \in \mathcal{T}_{h}\right\}$.

Lemma 5.1. For each $K \in \mathcal{T}_{h}$ and $F \in \mathcal{E}_{h}^{o}$, let $\psi_{K}$ and $\psi_{F}$ be defined as above. Then

$$
\begin{aligned}
& \psi_{K}=\nabla \psi_{K} \cdot \boldsymbol{n}=0 \quad \text { on } \partial K, \quad \psi_{F}=\nabla \psi_{F} \cdot \boldsymbol{n}=0 \quad \text { on the boundary of } w_{F}, \\
& \|v\|_{0, p, K} \lesssim\left\|v \psi_{K}^{\frac{1}{p}}\right\|_{0, p, K}, \quad\|w\|_{0, p, F} \lesssim\left\|w \psi_{F}^{\frac{1}{p}}\right\|_{0, p, F},
\end{aligned}
$$

for $p \in(1, \infty), v \in \mathcal{P}^{j}(K)$ and $w \in \mathcal{P}^{j}(F)$, where $j$ is a nonnegative integer.

Proof. The result (5.1) can be obtained directly by the definitions of $\psi_{K}$ and $\psi_{F}$, and the approximation result (5.2) can be derived by Lemma 3 from [20].

Lemma 5.2. Let $u$ and $\left(u_{h}, \widehat{u}_{h}\right)$ be the solutions of problems (2.1) and (2.2). Then we have

$$
h_{K}^{2}\left\|\Delta u_{h}\right\|_{0, K} \lesssim\left\|u-u_{h}\right\|_{0, K}, \quad \forall K \in \mathcal{T}_{h},
$$

and

$$
h_{K}\left\|\Delta u_{h}\right\|_{0, p, K} \lesssim\left\|\nabla\left(u-u_{h}\right)\right\|_{0, p, K}
$$

for $p \in\left(P^{\Omega}, 2\right)$. 
Proof. Let $v_{h}=\psi_{K} \Delta u_{h}$, by (5.1), (5.2) and inverse estimate (2.4) we arrive at

$$
\begin{aligned}
\left\|\Delta u_{h}\right\|_{0, K}^{2} & \lesssim\left(\Delta u_{h}, v_{h}\right)_{K}=\left(\Delta\left(u_{h}-u\right), v_{h}\right)_{K} \\
& =\left(u_{h}-u, \Delta v_{h}\right)_{K} \lesssim\left\|u-u_{h}\right\|_{0, K} h_{K}^{-2}\left\|v_{h}\right\|_{0, K} \\
& \lesssim h_{K}^{-2}\left\|u-u_{h}\right\|_{0, K}\left\|\Delta u_{h}\right\|_{0, K} .
\end{aligned}
$$

Hence the estimate (5.3) can be obtained by (5.5).

Similarly, let $v_{h}=\psi_{K} \Delta u_{h}$, we have

$$
\begin{aligned}
\left\|\Delta u_{h}\right\|_{0, K}^{2} & \lesssim\left(\Delta\left(u_{h}-u\right), v_{h}\right)_{K} \\
& =\left(\nabla\left(u_{h}-u\right), \nabla v_{h}\right)_{K} \lesssim\left\|\nabla\left(u-u_{h}\right)\right\|_{0, p, K} h_{K}^{-1}\left\|v_{h}\right\|_{0, p^{\prime}, K} \\
& \lesssim h_{K}^{-1} h_{K}^{\frac{d}{p^{\prime}}-\frac{d}{2}}\left\|\nabla\left(u-u_{h}\right)\right\|_{0, p, K}\left\|\Delta u_{h}\right\|_{0, K} .
\end{aligned}
$$

So we can obtain the error estimate (5.4) by (5.6) and inverse estimate (2.4).

For the case of $x_{0}$ is not a node of the partition $\mathcal{T}_{h}$, we know that the error estimators $\eta_{K}$ and $\zeta_{K, s}$ include an additional term. In order to control this term, we define a cutoff function $B_{x_{0}}$ for the element $K \in T_{x_{0}}$ such that

$$
\begin{aligned}
& 0 \leq B_{x_{0}} \leq 1 \quad \forall x \in \Omega, \\
& B_{x_{0}}=1 \quad \forall x \in \Omega:\left|x-x_{0}\right| \leq \frac{t}{4}, \\
& B_{x_{0}}=0 \quad \forall x \in \Omega:\left|x-x_{0}\right| \geq \frac{3 t}{4}, \\
& \left|B_{x_{0}}\right|_{m, \infty, w_{K}} \lesssim t^{-m} \quad m=1,2,
\end{aligned}
$$

where $w_{K}:=\bigcup\left\{K^{\prime} \in \mathcal{T}_{h}: K \cap K^{\prime} \neq \emptyset\right\}$, and $t$ denotes the distance of $x_{0}$ to the boundary of $w_{K}$. Note that the cutoff function has been used in [2]. Then using the fact that $h_{K} \lesssim t$ and (5.10), we have

$$
\left|B_{x_{0}}\right|_{m, p^{\prime}, w_{K}} \lesssim t^{-m} h_{K}^{d / p^{\prime}} \lesssim h_{K}^{d / p^{\prime}-m}, \quad m=1,2,1 \leq p^{\prime} \leq \infty .
$$

Lemma 5.3. For any $K \in T_{x_{0}}$, let $B_{x_{0}}$ and $w_{K}$ be defined as above. Then we have

$$
h_{K}^{2-\frac{d}{2}} \lesssim\left\|u-u_{h}\right\|_{0, w_{K}}+\sum_{K^{\prime} \in w_{K}} \tau_{0} h_{K^{\prime}}^{\frac{1}{2}}\left\|u_{h}-\widehat{u}_{h}\right\|_{0, \partial K^{\prime}},
$$

and

$$
h_{K}^{\frac{2}{p}-1} \lesssim\left\|\nabla\left(u-u_{h}\right)\right\|_{0, p, w_{K}}+\sum_{K^{\prime} \in w_{K}} \tau_{0} h_{K^{\prime}}^{\frac{1}{p}-1}\left\|u_{h}-\widehat{u}_{h}\right\|_{0, p, \partial K^{\prime}}
$$

for $p \in\left(P^{\Omega}, 2\right)$.

Proof. Using (4.1), the definition of $B_{x_{0}}$ and (5.11), we can infer that

$$
\begin{aligned}
1= & B_{x_{0}}\left(x_{0}\right)=\left(\nabla\left(u-u_{h}\right), \nabla B_{x_{0}}\right)_{\mathcal{T}_{h}}+\left(\nabla u_{h}, \nabla B_{x_{0}}\right)_{\mathcal{T}_{h}} \\
= & -\left(u-u_{h}, \Delta B_{x_{0}}\right)_{w_{K}}-\left(\Delta u_{h}, B_{x_{0}}\right)_{w_{K}} \\
& -\left\langle u_{h}, \nabla B_{x_{0}} \cdot \mathbf{n}\right\rangle_{\partial w_{K}}+\left\langle\nabla u_{h} \cdot \mathbf{n}, B_{x_{0}}\right\rangle_{\partial w_{K}} \\
\lesssim & \sum_{K^{\prime} \in w_{K}}\left(\left\|u-u_{h}\right\|_{0, K^{\prime}} h_{K}^{d / 2-2}+\left\|\Delta u_{h}\right\|_{0, K^{\prime}} h_{K}^{d / 2}\right. \\
& \left.+\tau_{0}\left\|u_{h}-\widehat{u}_{h}\right\|_{0, \partial K^{\prime}} h_{K}^{(d-1) / 2-1}\right),
\end{aligned}
$$


where $\partial w_{K}:=\left\{\partial K^{\prime}: K^{\prime} \in w_{K}\right\}$. Hence we can get (5.12) by using (5.14) and (5.3).

Similarly, we have

$$
\begin{aligned}
1= & \left(\nabla\left(u-u_{h}\right), \nabla B_{x_{0}}\right)_{w_{K}}-\left(\Delta u_{h}, B_{x_{0}}\right)_{w_{K}}+\left\langle\nabla u_{h} \cdot \mathbf{n}, B_{x_{0}}\right\rangle_{\partial w_{K}} \\
\lesssim & \sum_{K^{\prime} \in w_{K}}\left(\left\|\nabla\left(u-u_{h}\right)\right\|_{0, p, K^{\prime}} h_{K}^{2 / p^{\prime}-1}+\left\|\Delta u_{h}\right\|_{0, p, K^{\prime}} h_{K}^{2 / p^{\prime}}\right. \\
& \left.+\tau_{0}\left\|u_{h}-\widehat{u}_{h}\right\|_{0, p, \partial K^{\prime}} h_{K}^{1 / p^{\prime}-1}\right) .
\end{aligned}
$$

So we can obtain (5.13) by combining (5.15) and (5.4).

Obviously, the remaining task is to bound the error estimator $\left\|u_{h}-\widehat{u}_{h}\right\|_{0, p, \partial K}$. But, seemingly, it is not an easy task. First of all, we show the following relationships.

Lemma 5.4. Let $\left(u_{h}, \widehat{u}_{h}\right)$ be the solution of problem (2.2). Then the following relationships hold

$$
\left\|\left[\nabla u_{h} \cdot \boldsymbol{n}\right]\right\|_{0, p, F}^{p}+\tau_{0}^{p} h_{F}^{-p}\left\|\left[u_{h}\right]\right\|_{0, p, F}^{p} \lesssim 2^{p-1}\left(\left\|\frac{\tau_{0}}{h_{F}}\left(u_{h}^{+}-\widehat{u}_{h}\right)\right\|_{0, p, F}^{p}+\left\|\frac{\tau_{0}}{h_{F}}\left(u_{h}^{-}-\widehat{u}_{h}\right)\right\|_{0, p, F}^{p}\right),
$$

and

$$
\left\|\frac{\tau_{0}}{h_{F}}\left(u_{h}^{+}-\widehat{u}_{h}\right)\right\|_{0, p, F}^{p}+\left\|\frac{\tau_{0}}{h_{F}}\left(u_{h}^{-}-\widehat{u}_{h}\right)\right\|_{0, p, F}^{p} \lesssim 2^{p-1}\left(\left\|\left[\nabla u_{h} \cdot \boldsymbol{n}\right]\right\|_{0, p, F}^{p}+\tau_{0}^{p} h_{F}^{-p}\left\|\left[u_{h}\right]\right\|_{0, p, F}^{p}\right),
$$

for $1 \leq p<\infty$ and $\mathcal{E}_{h}^{o} \ni F=\overline{K^{+}} \cap \overline{K^{-}}$.

Proof. To prove (5.16) and (5.17), we only need to prove the following results:

$$
\begin{gathered}
\left|\left[\nabla u_{h} \cdot \mathbf{n}\right]\right|^{p}+\tau_{0}^{p} h_{F}^{-p}\left|\left[u_{h}\right]\right|^{p} \lesssim 2^{p-1}\left(\left|\frac{\tau_{0}}{h_{F}}\left(u_{h}^{+}-\widehat{u}_{h}\right)\right|^{p}+\left|\frac{\tau_{0}}{h_{F}}\left(u_{h}^{-}-\widehat{u}_{h}\right)\right|^{p}\right), \\
\left|\frac{\tau_{0}}{h_{F}}\left(u_{h}^{+}-\widehat{u}_{h}\right)\right|^{p}+\left|\frac{\tau_{0}}{h_{F}}\left(u_{h}^{-}-\widehat{u}_{h}\right)\right|^{p} \lesssim 2^{p-1}\left(\left|\left[\nabla u_{h} \cdot \mathbf{n}\right]\right|^{p}+\tau_{0}^{p} h_{F}^{-p}\left|\left[u_{h}\right]\right|^{p}\right) .
\end{gathered}
$$

By using (4.1) and the definition of the jump $\left[u_{h}\right]$, we yield

$$
\left|\left[\nabla u_{h} \cdot \mathbf{n}\right]\right|+\tau_{0} h_{F}^{-1}\left|\left[u_{h}\right]\right| \lesssim\left|\frac{\tau_{0}}{h_{F}}\left(u_{h}^{+}-\widehat{u}_{h}\right)\right|+\left|\frac{\tau_{0}}{h_{F}}\left(u_{h}^{-}-\widehat{u}_{h}\right)\right| .
$$

Hence we can get (5.18) by the above inequality.

On the other hand, by using the triangle inequality, we yield

$$
\begin{aligned}
\left|\frac{\tau_{0}}{h_{F}}\left(u_{h}^{+}-\widehat{u}_{h}\right)\right| & =\frac{1}{2}\left|\tau_{0} h_{F}^{-1}\left[u_{h}\right]+\frac{\tau_{0}}{h_{F}}\left(u_{h}^{+}-\widehat{u}_{h}\right)+\frac{\tau_{0}}{h_{F}}\left(u_{h}^{-}-\widehat{u}_{h}\right)\right| \\
& \leq \frac{1}{2} \tau_{0} h_{F}^{-1}\left|\left[u_{h}\right]\right|+\frac{1}{2}\left|\left[\nabla u_{h} \cdot \mathbf{n}\right]\right|
\end{aligned}
$$

and

$$
\left|\frac{\tau_{0}}{h_{F}}\left(u_{h}^{-}-\widehat{u}_{h}\right)\right| \leq \frac{1}{2} \tau_{0} h_{F}^{-1}\left|\left[u_{h}\right]\right|+\frac{1}{2}\left|\left[\nabla u_{h} \cdot \mathbf{n}\right]\right| .
$$

Therefore the estimate (5.19) can be derived by the above two inequalities.

Now we are going to bound the error estimator $\left\|\left[\nabla u_{h} \cdot \mathbf{n}\right]\right\|_{0, p, F}^{p}$ for $F \in \mathcal{E}_{h}^{o}$. 
Lemma 5.5. Let $u$ and $\left(u_{h}, \widehat{u}_{h}\right)$ be the solutions of problems (1.1) and (2.2). Then for each $F \in \mathcal{E}_{h}^{o}$, we have

$$
h_{F}^{\frac{3}{2}}\left\|\left[\nabla u_{h} \cdot \boldsymbol{n}\right]\right\|_{0, F} \lesssim\left\|u-u_{h}\right\|_{0, w_{F}}+h_{F}^{\frac{1}{2}}\left\|\left[u_{h}\right]\right\|_{0, F},
$$

and

$$
h_{F}^{\frac{1}{p}}\left\|\left[\nabla u_{h} \cdot \boldsymbol{n}\right]\right\|_{0, p, F} \lesssim\left\|\nabla\left(u-u_{h}\right)\right\|_{0, p, w_{F}}
$$

for $p \in\left(P^{\Omega}, 2\right)$.

Proof. Let $P_{F}: L^{\infty}(F) \rightarrow L^{\infty}\left(w_{F}\right)$ be a continuation operator [35] such that

$$
\left\|P_{F} w\right\|_{0, w_{F}} \lesssim h_{F}^{\frac{1}{2}}\|w\|_{0, F}, \quad \forall w \in \mathcal{P}^{j}(F),
$$

for any nonnegative integer $j$. Let $v=\psi_{F}\left(P_{F}\left[\nabla u_{h} \cdot \mathbf{n}\right]\right)$.

By the trace inequality, the inverse estimate (2.4), Lemma 5.1 and (5.22), we can get

$$
\begin{aligned}
\left\|\left[\nabla u_{h} \cdot \mathbf{n}\right]\right\|_{0, F}^{2} \lesssim & \left\langle\left[\nabla u_{h} \cdot \mathbf{n}\right], v\right\rangle_{F}=\int_{w_{F}} \nabla \cdot\left(v \nabla u_{h}\right) \\
= & \left(\Delta u_{h}, v\right)_{w_{F}}+\left(\nabla v, \nabla u_{h}\right)_{w_{F}} \\
\leq & \left\|\Delta u_{h}\right\|_{0, w_{F}}\|v\|_{0, w_{F}}-\left(\Delta v, u_{h}-u\right)_{w_{F}}+\left\langle\nabla v \cdot \mathbf{n},\left[u_{h}\right]\right\rangle_{F} \\
\lesssim & \left\|\left[\nabla u_{h} \cdot \mathbf{n}\right]\right\|_{0, F}\left(\left\|\Delta u_{h}\right\|_{0, w_{F}} h_{F}^{\frac{1}{2}}+h_{F}^{-\frac{3}{2}}\left\|u-u_{h}\right\|_{0, w_{F}}\right. \\
& \left.+h_{F}^{-1}\left\|\left[u_{h}\right]\right\|_{0, F}\right) .
\end{aligned}
$$

Hence the approximation (5.20) can be obtained by (5.3) and the above estimate.

Similarly, we have

$$
\begin{aligned}
\left\|\left[\nabla u_{h} \cdot \mathbf{n}\right]\right\|_{0, F}^{2} & \lesssim\left(\Delta u_{h}, v\right)_{w_{F}}+\left(\nabla v, \nabla\left(u_{h}-u\right)\right)_{w_{F}} \\
& \lesssim\left\|\left[\nabla u_{h} \cdot \mathbf{n}\right]\right\|_{0, F}\left(h_{F}^{\frac{d}{p^{\prime}}-\frac{d}{2}+\frac{1}{2}}\left\|\Delta u_{h}\right\|_{0, p, w_{F}}+h_{F}^{\frac{d}{p^{\prime}}-\frac{d}{2}-\frac{1}{2}}\left\|\nabla\left(u-u_{h}\right)\right\|_{0, p, w_{F}}\right) .
\end{aligned}
$$

Therefore, we can derive (5.21) by (5.4), the inverse estimate (2.4) and (5.23).

Combining Lemmas (4.2), (4.4), (5.2), (5.3), (5.4) and (5.5) we can get the following theorem.

Theorem 5.6. Let $u$ and $\left(u_{h}, \widehat{u}_{h}\right)$ be the solutions of problems (2.1) and (2.2). Let $\widetilde{u}_{h}$ be the Oswald interpolation of $u_{h}$.

(i) If the domain $\Omega$ is convex, we have

$$
\begin{aligned}
& \left\|u-u_{h}\right\|_{0, \Omega}+\left\|u-\widetilde{u}_{h}\right\|_{0, \Omega} \lesssim\left(\sum_{K \in \mathcal{T}_{h}} \eta_{K}^{2}\right)^{1 / 2}, \\
& \left(\sum_{K \in \mathcal{T}_{h}} \eta_{K}^{2}\right)^{1 / 2} \lesssim \tau_{0}\left(\left\|u-u_{h}\right\|_{0, \Omega}+\left\|u-\widetilde{u}_{h}\right\|_{0, \Omega}\right),
\end{aligned}
$$

(ii) If $d=2$ and the domain $\Omega$ is a Lipschitz polygon, we have

$$
\begin{aligned}
& \left\|\nabla\left(u-u_{h}\right)\right\|_{0, p, \mathcal{T}_{h}}+\left(\sum_{F \in \mathcal{E}_{h}} \tau_{0}^{p} h_{F}^{1-p}\left\|\left[u_{h}-\widetilde{I} u\right]\right\|_{0, p, F}^{p}\right)^{1 / p} \lesssim\left(\sum_{K \in \mathcal{T}_{h}} \zeta_{K, p}^{p}\right)^{1 / p} \\
& \left(\sum_{K \in \mathcal{T}_{h}} \zeta_{K, p}^{p}\right)^{1 / p} \lesssim\left\|\nabla\left(u-u_{h}\right)\right\|_{0, p, \Omega}+\left(\sum_{F \in \mathcal{E}_{h}} \tau_{0}^{p} h_{F}^{1-p}\left\|\left[u_{h}-\widetilde{I} u\right]\right\|_{0, p, F}^{p}\right)^{1 / p}
\end{aligned}
$$

for $p \in\left(P^{\Omega}, 2\right)$, where $P^{\Omega}=\max \left\{1,2 /\left(1+\frac{\pi}{\theta}\right)\right\}$ and $\theta$ is the largest inner angle of the domain $\Omega$. 
Proof. By using Oswald interpolation error estimate (2.11), we infer that

$$
\begin{aligned}
\left\|u-\widetilde{u}_{h}\right\|_{0, \Omega} & \leq\left\|u-u_{h}\right\|_{0, \Omega}+\left(\sum_{K \in \mathcal{T}_{h}}\left\|u_{h}-\widetilde{u}_{h}\right\|_{0, K}^{2}\right)^{1 / 2} \\
& \lesssim\left\|u-u_{h}\right\|_{0, \Omega}+\left(\sum_{F \in \mathcal{E}_{h}^{o}} h_{F}\left\|\left[u_{h}\right]\right\|_{0, F}^{2}+\sum_{F \in \mathcal{E}_{h}^{\partial}} h_{F}\left\|u_{h}\right\|_{0, F}^{2}\right)^{1 / 2} \\
& \lesssim\left\|u-u_{h}\right\|_{0, \Omega}+\left(\sum_{K \in \mathcal{T}_{h}} h_{K}\left\|u_{h}-\widehat{u}_{h}\right\|_{0, \partial K}^{2}\right)^{1 / 2} .
\end{aligned}
$$

Hence we can get (5.24) by combining (4.3) and (5.28).

In virtue of the results introduced in Lemmas 5.2, 5.3, 5.4 and 5.5, we yield

$$
\left(\sum_{K \in \mathcal{T}_{h}} \eta_{K}^{2}\right)^{1 / 2} \lesssim\left\|u-u_{h}\right\|_{0, \Omega}+\left(\sum_{F \in \mathcal{E}_{h}^{o}} \tau_{0}^{2} h_{F}\left\|\left[u_{h}\right]\right\|_{0, F}^{2}+\sum_{F \in \mathcal{E}_{h}^{\partial}} \tau_{0}^{2} h_{F}\left\|u_{h}\right\|_{0, F}^{2}\right)^{1 / 2}
$$

and

$$
\begin{aligned}
\left(\sum_{K \in \mathcal{T}_{h}} \zeta_{K, p}^{p}\right)^{1 / p} & \lesssim\left\|\nabla\left(u-u_{h}\right)\right\|_{0, p, \mathcal{T}_{h}}+\left(\sum_{F \in \mathcal{E}_{h}^{o}} \tau_{0}^{p} h_{F}^{1-p}\left\|\left[u_{h}\right]\right\|_{0, p, F}^{p}+\sum_{F \in \mathcal{E}_{h}^{\partial}} \tau_{0}^{p} h_{F}^{1-p}\left\|u_{h}\right\|_{0, p, F}^{p}\right)^{1 / p} \\
& =\left\|\nabla\left(u-u_{h}\right)\right\|_{0, p, \mathcal{T}_{h}}+\left(\sum_{F \in \mathcal{E}_{h}} \tau_{0}^{p} h_{F}^{1-p}\left\|\left[u_{h}-\widetilde{I}_{u}\right]\right\|_{0, p, F}^{p}\right)^{1 / p}
\end{aligned}
$$

Obviously, the approximation result (5.30) is the same as (5.27). Moreover the inverse estimate (2.4) and trace inequality (2.5) result in

$$
\begin{aligned}
h_{F}^{\frac{1}{2}}\left\|\left[u_{h}\right]\right\|_{0, F} & =h_{F}^{\frac{1}{2}}\left\|\left[u_{h}-\widetilde{u}_{h}\right]\right\|_{0, F} \leq h_{F}^{\frac{1}{2}}\left\|u_{h}^{+}-\widetilde{u}_{h}\right\|_{0, F}+h_{F}^{\frac{1}{2}}\left\|u_{h}^{-}-\widetilde{u}_{h}\right\|_{0, F} \\
& \lesssim\left\|u_{h}^{+}-\widetilde{u}_{h}\right\|_{0, K^{+}}+\left\|u_{h}^{-}-\widetilde{u}_{h}\right\|_{0, K^{-}},
\end{aligned}
$$

for any $\mathcal{E}_{h}^{o} \ni F=\overline{K^{+}} \cap \overline{K^{-}}$. Hence

$$
\begin{aligned}
\left(\sum_{F \in \mathcal{E}_{h}^{o}} \tau_{0}^{2} h_{F}\left\|\left[u_{h}\right]\right\|_{0, F}^{2}+\sum_{F \in \mathcal{E}_{h}^{o}} \tau_{0}^{2} h_{F}\left\|u_{h}\right\|_{0, F}^{2}\right)^{1 / 2} & \lesssim \tau_{0}\left\|u_{h}-\widetilde{u}_{h}\right\|_{0, \Omega} \\
& \lesssim \tau_{0}\left(\left\|u-u_{h}\right\|_{0, \Omega}+\left\|u-\widetilde{u}_{h}\right\|_{0, \Omega}\right),
\end{aligned}
$$

which, together with (5.29), derives the approximation result (5.25).

Finally the approximation result (5.26) can be obtained by Lemma 4.4 and the fact that

$$
\left(\sum_{F \in \mathcal{E}_{h}} \tau_{0}^{p} h_{F}^{1-p}\left\|\left[u_{h}-\widetilde{I} u\right]\right\|_{0, p, F}^{p}\right)^{1 / p} \lesssim\left(\sum_{K \in \mathcal{T}_{h}} \tau_{0}^{p} h_{K}^{1-p}\left\|u_{h}-\widehat{u}_{h}\right\|_{0, p, K}^{p}\right)^{1 / p}
$$

\section{NUMERICAL EXPERIMENTS}

In this section, some numerical experiments are provided to validate the theoretical analysis. For the adaptive HDG algorithm designed by the obtained a posteriori error estimators, we use the following marking strategy

$$
\sum_{K \in \mathcal{M}} \xi_{K} \geq \gamma \xi
$$


TABLE 1 . The convergence history of $\left\|u-u_{h}\right\|_{0, \Omega}$ for HDG scheme (2.2) for $k=1$.

\begin{tabular}{ccccccc}
\hline \hline$h$ & $\left\|e_{u}\right\|_{0, \Omega}\left(\tau_{0}=15\right)$ & Order & $\left\|e_{u}\right\|_{0, \Omega}\left(\tau_{0}=25\right)$ & Order & $\left\|e_{u}\right\|_{0, \Omega}\left(\tau_{0}=100\right)$ & Order \\
\hline$\frac{1}{4}$ & $1.4376 \mathrm{e}-2$ & & $1.3591 \mathrm{e}-2$ & & $1.4455 \mathrm{e}-2$ & \\
$\frac{1}{8}$ & $7.1722 \mathrm{e}-3$ & 1.003 & $6.7986 \mathrm{e}-3$ & 0.999 & $7.3180 \mathrm{e}-3$ & 0.982 \\
$\frac{1}{16}$ & $3.5832 \mathrm{e}-3$ & 1.001 & $3.3979 \mathrm{e}-3$ & 1.001 & $3.6712 \mathrm{e}-3$ & 0.995 \\
$\frac{1}{32}$ & $1.7912 \mathrm{e}-3$ & 1.000 & $1.6987 \mathrm{e}-3$ & 1.000 & $1.8371 \mathrm{e}-3$ & 0.999 \\
$\frac{1}{64}$ & $8.9554 \mathrm{e}-4$ & 1.000 & $8.4934 \mathrm{e}-4$ & 1.000 & $9.1873 \mathrm{e}-4$ & 1.000 \\
$\frac{1}{128}$ & $4.4777 \mathrm{e}-4$ & 1.000 & $4.2467 \mathrm{e}-4$ & 1.000 & $4.5939 \mathrm{e}-4$ & 1.000 \\
$\frac{1}{256}$ & $2.2388 \mathrm{e}-4$ & 1.000 & $2.1233 \mathrm{e}-4$ & 1.000 & $2.2970 \mathrm{e}-4$ & 1.000 \\
\hline
\end{tabular}

TABLE 2. The convergence history of $\left\|u-u_{h}\right\|_{0, \Omega}$ for HDG scheme (2.2) for $k=2$.

\begin{tabular}{ccccccc}
\hline \hline$h$ & $\left\|e_{u}\right\|_{0, \Omega}\left(\tau_{0}=15\right)$ & Order & $\left\|e_{u}\right\|_{0, \Omega}\left(\tau_{0}=25\right)$ & Order & $\left\|e_{u}\right\|_{0, \Omega}\left(\tau_{0}=100\right)$ & Order \\
\hline$\frac{1}{4}$ & $3.6253 \mathrm{e}-2$ & & $9.1561 \mathrm{e}-3$ & & $6.9934 \mathrm{e}-3$ & \\
$\frac{1}{8}$ & $1.8224 \mathrm{e}-2$ & 0.992 & $4.5782 \mathrm{e}-3$ & 1.000 & $3.4990 \mathrm{e}-3$ & 0.999 \\
$\frac{1}{16}$ & $9.1121 \mathrm{e}-3$ & 1.000 & $2.2891 \mathrm{e}-3$ & 1.000 & $1.7495 \mathrm{e}-3$ & 1.000 \\
$\frac{1}{32}$ & $4.5560 \mathrm{e}-3$ & 1.000 & $1.1446 \mathrm{e}-3$ & 1.000 & $8.7477 \mathrm{e}-4$ & 1.000 \\
$\frac{1}{64}$ & $2.2780 \mathrm{e}-3$ & 1.000 & $5.7228 \mathrm{e}-4$ & 1.000 & $4.3738 \mathrm{e}-4$ & 1.000 \\
$\frac{1}{128}$ & $1.1390 \mathrm{e}-3$ & 1.000 & $2.8614 \mathrm{e}-4$ & 1.000 & $2.1869 \mathrm{e}-4$ & 1.000 \\
$\frac{1}{256}$ & $5.6950 \mathrm{e}-4$ & 1.000 & $1.4307 \mathrm{e}-4$ & 1.000 & $1.0935 \mathrm{e}-4$ & 1.000 \\
\hline
\end{tabular}

to select the marking set $\mathcal{M}$, where $\gamma \in(0,1], \xi=\eta_{2}^{2}$ or $\zeta_{p}^{p}$, and $\xi_{K}$ is the restriction of $\xi$ on the element $K \in \mathcal{T}_{h}$. Here $\eta_{2}^{2}=\sum_{K \in \mathcal{T}_{h}} \eta_{K}^{2}$ and $\zeta_{p}^{p}=\sum_{K \in \mathcal{T}_{h}} \zeta_{K}^{p}$. We refine the marking set $\mathcal{M}$ by bisections to generate a new mesh.

Note here that the figure of convergence history is plotted in log-log coordinates in this section. Moreover we set $e_{u}=u-u_{h}$ and

$$
\begin{aligned}
& E=\left\|e_{u}\right\|_{0, \Omega}+\left\|u-\tilde{u}_{h}\right\|_{0, \Omega}, \\
& E_{2, s}=\left\|\nabla e_{u}\right\|_{0, s, \Omega}+\left(\sum_{F \in \mathcal{E}_{h}} \tau_{0}^{s} h_{F}^{1-s}\left\|\left[u_{h}\right]\right\|_{0, s, F}^{s}\right)^{1 / s} .
\end{aligned}
$$

Example 6.1. Based on the domain $\Omega=(0,1)^{2}$, we consider the problem $(1.1)$ with $x_{0}=(0.5,0.5)$. In this example, the Dirichlet boundary conditions are imposed so that the exact solution is given by

$$
u(\mathbf{x})=-\frac{1}{2 \pi} \log \left|\mathbf{x}-x_{0}\right| .
$$

We test this example under the constraint that $x_{0}$ be a vertex of all partitions.

In Tables 1 and 2, the convergence history and convergence order of the error $\left\|e_{u}\right\|_{0, \Omega}$ for different $\tau_{0}$ and $k$ are provided on uniform meshes. We find that the convergence rate $O(h)$ can be achieved. From the perspective of error, we find from Figure 1 that the best choice of $\tau_{0}$ may be 25 and 150 for $k=1$ and $k=2$. Furthermore, in Figure 2 and Table 3, the convergence histories of $\left\|u-u_{h}\right\|_{0, \Omega}$ for finite element methods (FEM) and HDG methods are presented for $k=1$. Obviously, involving a suitable choice of $\tau_{0}$, we can expect that the HDG methods of this paper are slightly better than the finite element methods.

From now on, we set $\tau_{0}=25$ and we test this example with adaptive HDG algorithm. The meshes and the corresponding surfaces of $u_{h}$, generated by $\eta_{2}$ and $\zeta_{1.5}$, are provided in Figures 3 and 5 for $k=1$ and $\gamma=0.2$. 

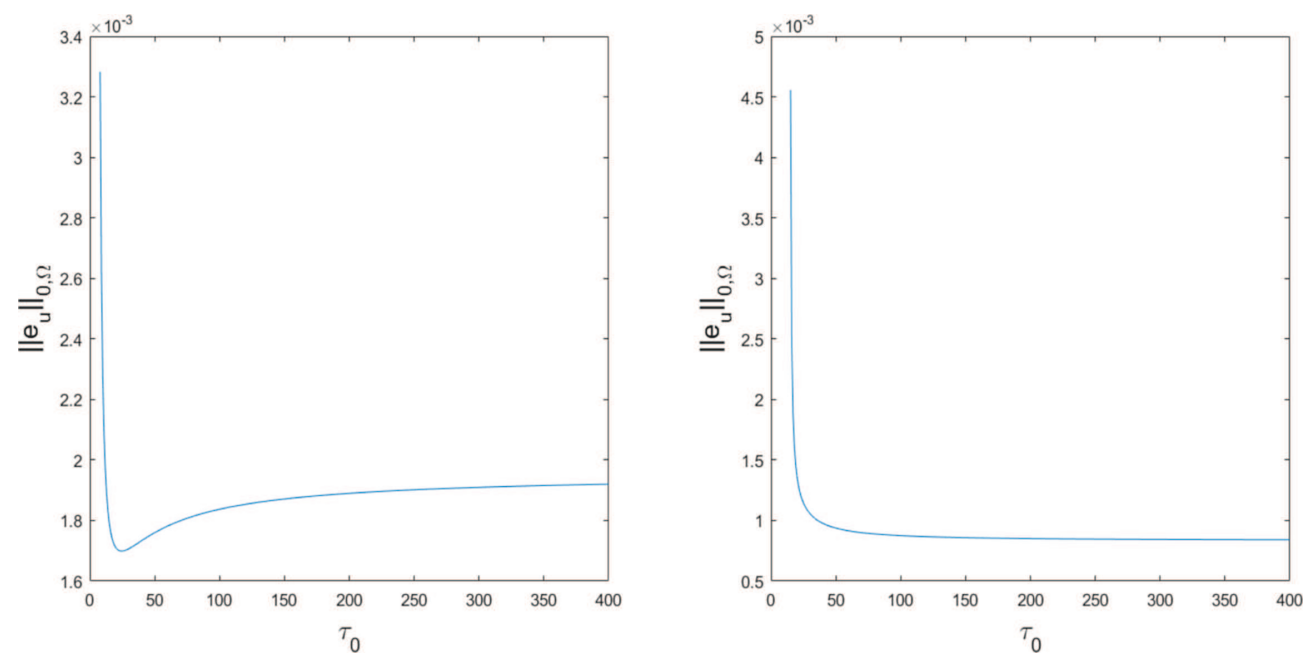

FiguRE 1. Left: The error $\left\|u-u_{h}\right\|_{0, \Omega}$ for different $\tau_{0}$ for $k=1$. Right: The error $\left\|u-u_{h}\right\|_{0, \Omega}$ for different $\tau_{0}$ for $k=2$. Here we set $h=\frac{1}{32}$.

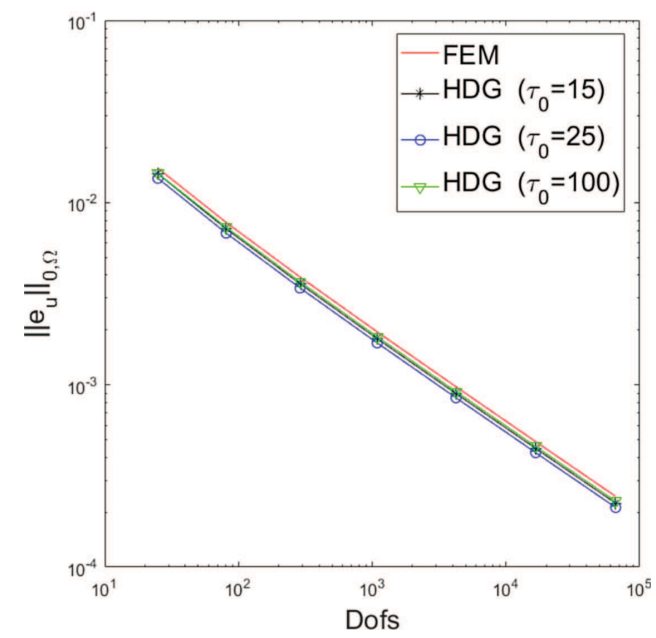

FiguRE 2. The convergence histories of $\left\|u-u_{h}\right\|_{0, \Omega}$ for finite element methods and HDG methods. Here we set $k=1$.

Obviously, the mesh nodes are concentrated around $x_{0}$. In Figure 4, we present the convergence histories of $\eta_{2}$ and $\left\|e_{u}\right\|_{0, \Omega}$ for different $k$ and $\gamma$. We observe from the left graph of Figure 4 that the convergence rate $O\left(N^{-(k+1) / 2}\right)$ can be obtained. From the right graph of Figure 4, we can see that for the same numerical accuracy the number of required vertices will be increased as $\gamma$ becomes large.

By a simple calculation, we have $P^{\Omega}=1$. Hence in the left graph of Figure 6, we give the convergence histories of $\zeta_{p}$ and $\left\|\nabla e_{u}\right\|_{0, p, \Omega}$ for $k=1, \gamma=0.2$ and $p=1.2,1.5,1.8$. The results show that these errors and estimators can obtain the convergence rate $O\left(N^{-1 / 2}\right)$. In the right graph of Figure 6 , we also plot the convergence histories of $\zeta_{1.5}$ and $\left\|\nabla e_{u}\right\|_{0,1.5, \Omega}$ for different $\gamma$. Obviously, the convergence rate decreases as $\gamma$ increases. 
TABLE 3 . The convergence history of $\left\|u-u_{h}\right\|_{0, \Omega}$ for finite element methods and HDG methods for $k=1$.

\begin{tabular}{ccccc}
\hline \hline$h$ & $\left\|e_{u}\right\|_{0, \Omega}(\mathrm{FEM})$ & Order & $\left\|e_{u}\right\|_{0, \Omega}\left(\mathrm{HDG}, \tau_{0}=25\right)$ & Order \\
\hline$\frac{1}{4}$ & $1.5426 \mathrm{e}-2$ & & $1.3591 \mathrm{e}-2$ & \\
$\frac{1}{8}$ & $7.7780 \mathrm{e}-3$ & 0.988 & $6.7986 \mathrm{e}-3$ & 0.999 \\
$\frac{1}{16}$ & $3.9028 \mathrm{e}-3$ & 0.995 & $3.3979 \mathrm{e}-3$ & 1.001 \\
$\frac{1}{32}$ & $1.9532 \mathrm{e}-3$ & 0.999 & $1.6987 \mathrm{e}-3$ & 1.000 \\
$\frac{1}{64}$ & $9.7683 \mathrm{e}-4$ & 1.000 & $8.4934 \mathrm{e}-4$ & 1.000 \\
$\frac{1}{128}$ & $4.8844 \mathrm{e}-4$ & 1.000 & $4.2467 \mathrm{e}-4$ & 1.000 \\
$\frac{1}{256}$ & $2.4422 \mathrm{e}-4$ & 1.000 & $2.2970 \mathrm{e}-4$ & 1.000 \\
\hline
\end{tabular}
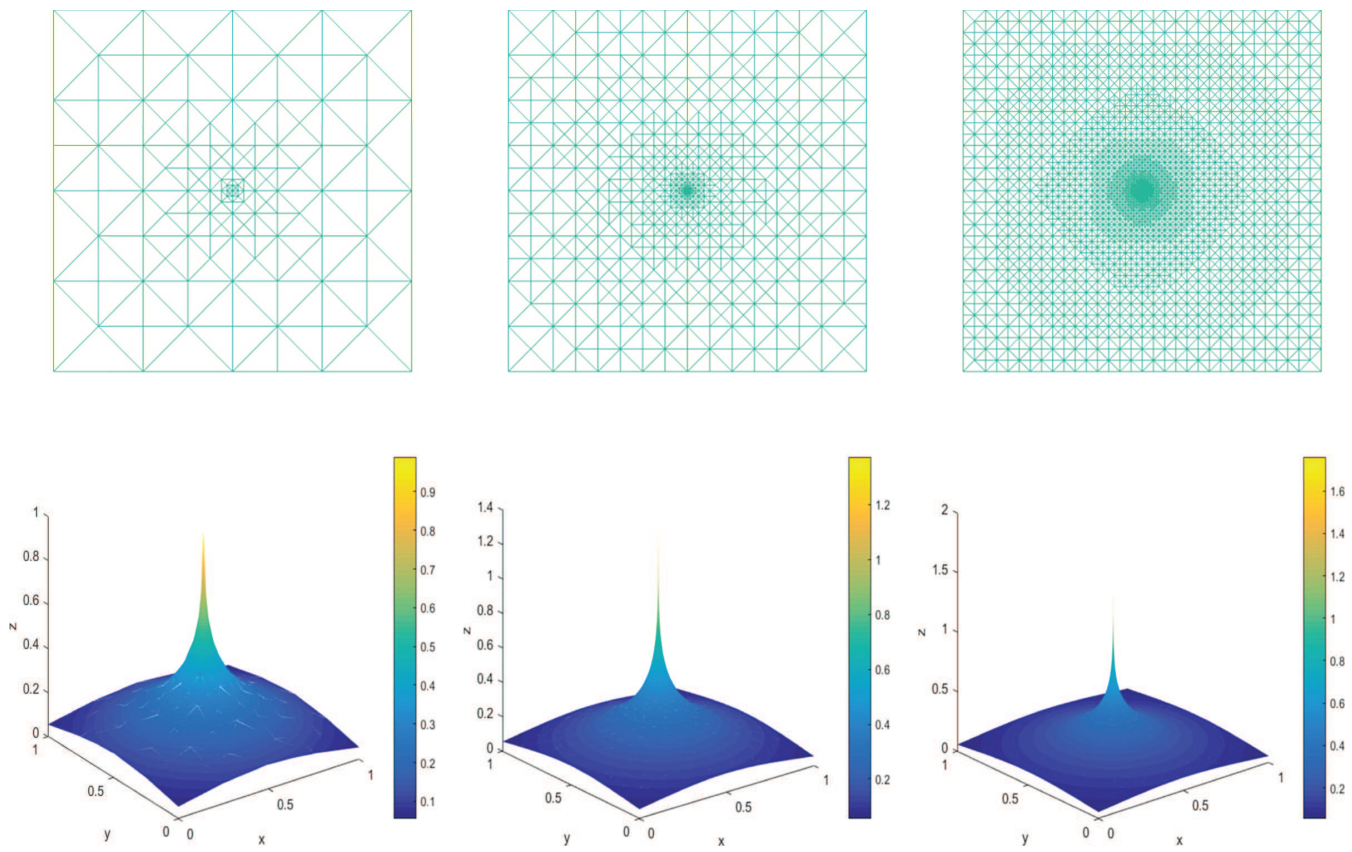

Figure 3 . Top: The adaptive meshes, generated by $\eta_{2}$, with 123, 636 and 3165 nodes. Bottom:

The corresponding surfaces of $u_{h}$. Here we set $k=1$ and $\gamma=0.2$.

Example 6.2. In this example, we consider the problem (1.1) in the L-shaped domain $\Omega=(-1,1)^{2} \backslash[0,1) \times$ $(-1,0]$ with $x_{0}=(0.5,0.5)$. The Dirichlet boundary conditions are imposed such that the exact solution is given by

$$
u(x)=-\frac{1}{2 \pi} \log \left|x-x_{0}\right|+|x|^{2 / 3} \sin \left(\frac{2 \vartheta}{3}\right),
$$

where $\vartheta \in\left(0, \frac{3 \pi}{2}\right)$ denotes the angle.

The initial mesh consists of 12 triangles. According to the definition of $P^{\Omega}$, we know that $P^{\Omega}=1.2$. Throughout this section, let $\tau_{0}=15$ and $x_{0}$ be a vertex of all partitions.

In Figure 7, the convergence histories of $\eta_{2}$ and $\left\|e_{u}\right\|_{0, \Omega}$ and the efficiency index $\eta_{2} / E$ are given. The convergence rate $O\left(N^{-(k+1) / 2}\right)$ can be obtained. In Figure 8, the adaptive meshes, generated by $\zeta_{1.3}$ and $\zeta_{1.5}$, after 

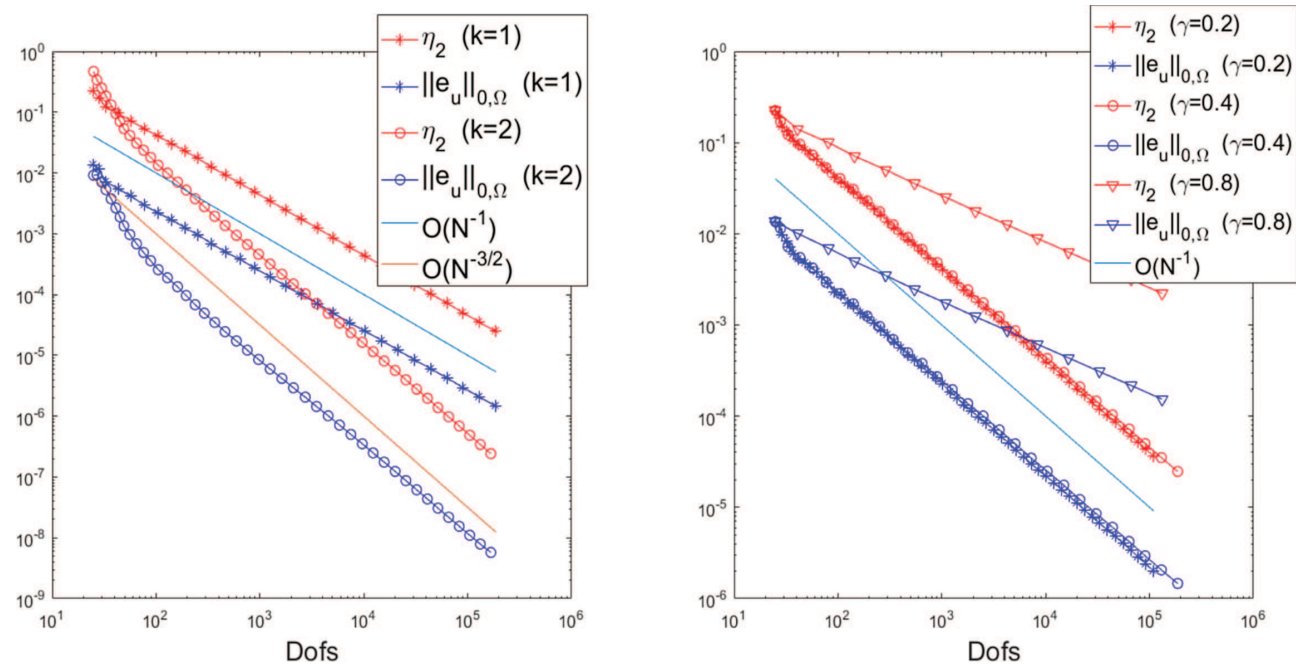

FiguRE 4. Left: The convergence histories of $\eta_{2}$ and $\|e\|_{0, \Omega}$ for $\gamma=0.4$ and $k=1,2$. Right: The convergence histories of $\eta_{2}$ and $\left\|e_{u}\right\|_{0, \Omega}$ for $k=1$ and $\gamma=0.2,0.4,0.8$.
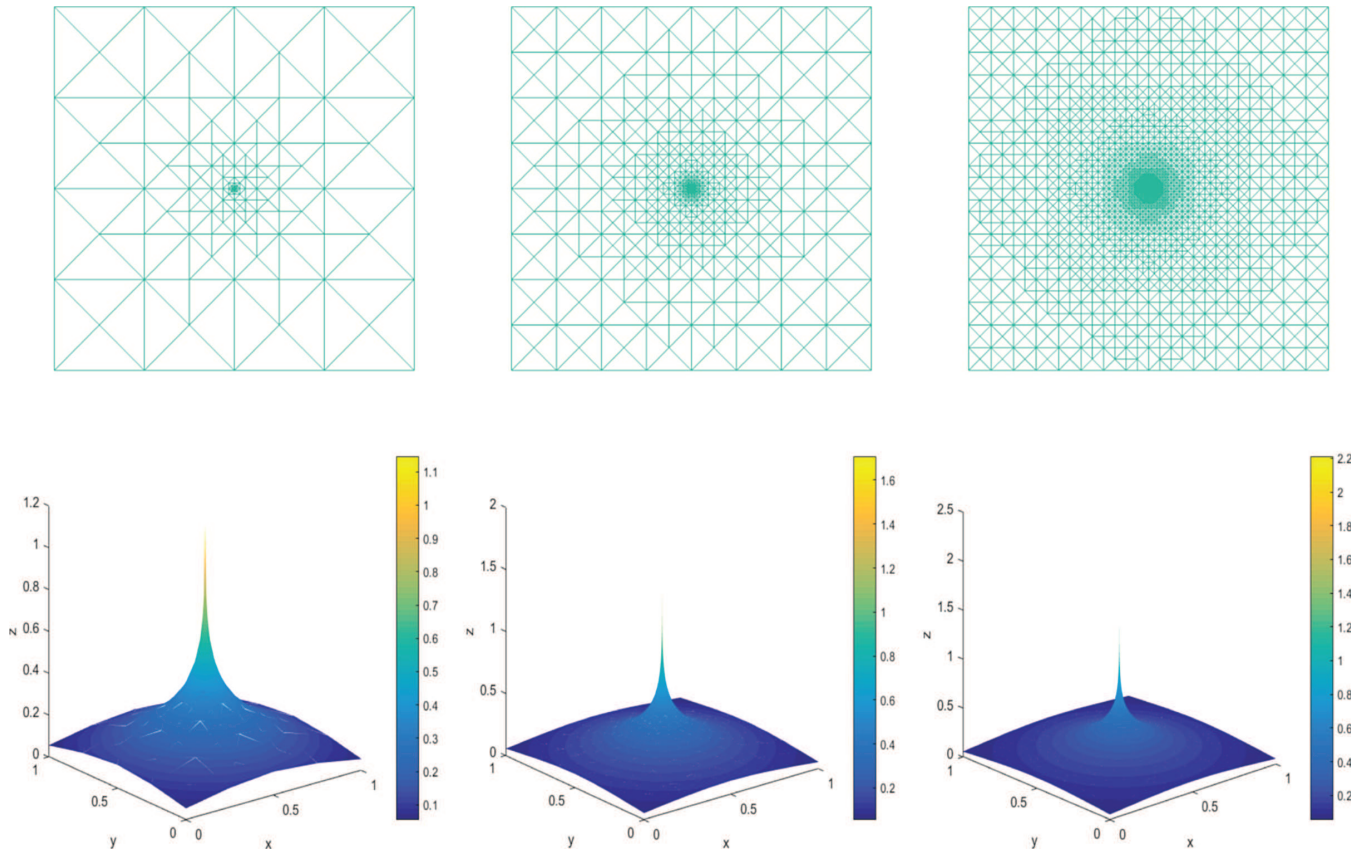

FiguRE 5. Top: The adaptive meshes, generated by $\zeta_{1.5}$, with 124, 686 and 3513 nodes. Bottom: The corresponding surfaces of $u_{h}$. Here we set $k=1$ and $\gamma=0.2$. 

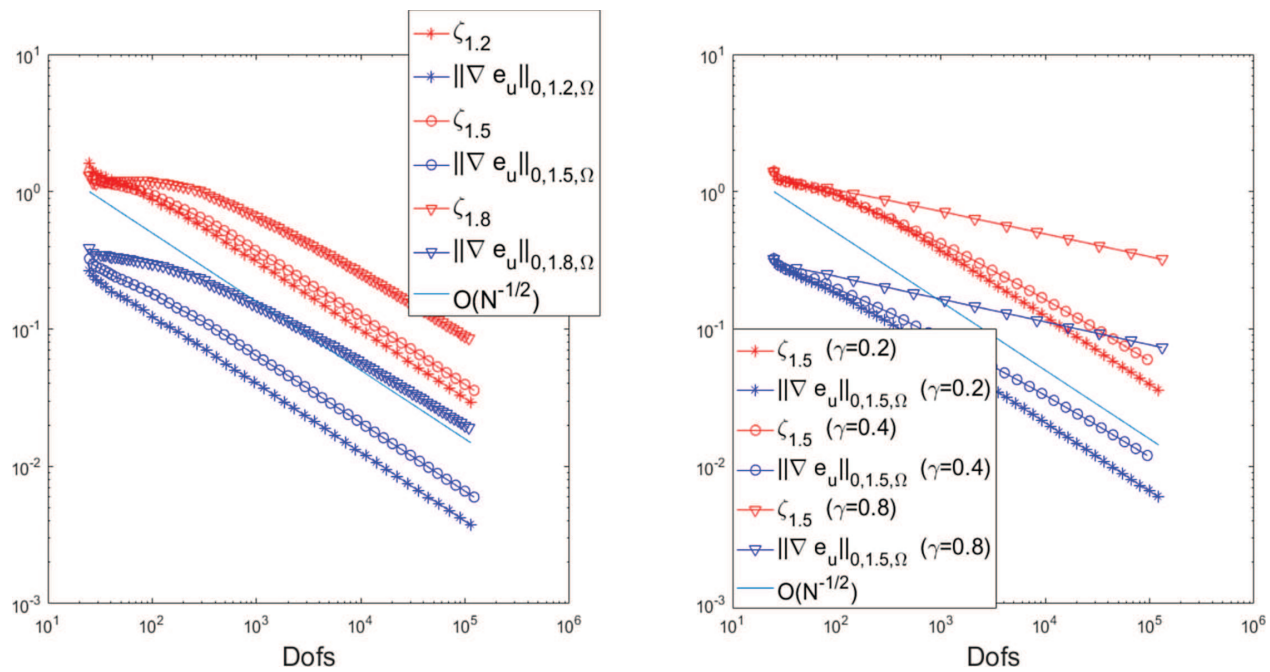

FiguRE 6. Left: The convergence histories for $\gamma=0.2$ and $k=1$. Right: The convergence histories for $k=1$ and $\gamma=0.2,0.4,0.8$.
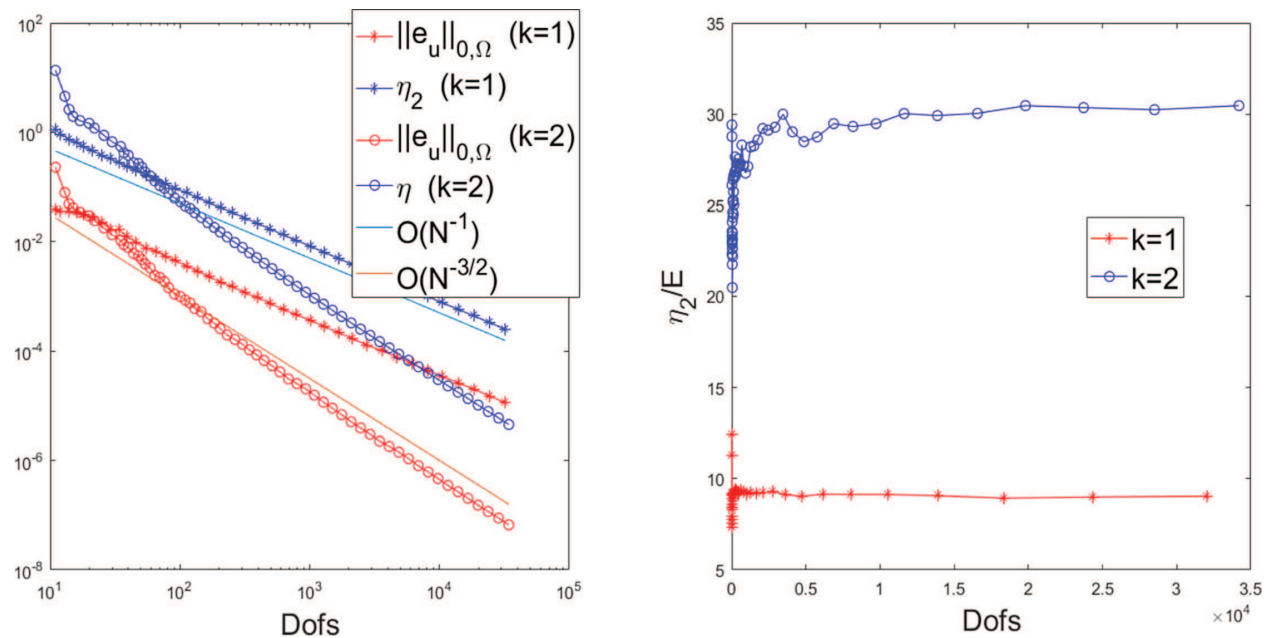

Figure 7. Left: The convergence histories of $\left\|e_{u}\right\|_{0, \Omega}$ and $\eta_{2}$ for $k=1$ and $k=2$. Right: The efficiency index $\eta_{2} / E$ for $k=1$ and $k=2$. Here we set $\gamma=0.3$.

13, 19 and 25 iterations are shown for $k=1$ and $\gamma=0.3$. We find that the mesh nodes are concentrated around the point $x_{0}$ and the reentrant corner. In Figure 9, the convergence histories of $\left\|\nabla e_{u}\right\|_{0, s, \Omega}, E_{2, s}$, and $\zeta_{s}$ are performed for $\gamma=0.3, k=1,2$ and $s=1.3,1.5$. The errors and estimators can all get the convergence rate $O\left(N^{-k / 2}\right)$.

Finally, we make a comparison between [2] and this paper for $k=1$ and $\gamma=0.3$. Note that the estimators and errors obtained in [2] are labeled by $\zeta_{s}^{\text {Ref }}$ and $e_{u}^{\text {Ref }}$. In Figure 10, the convergence histories are performed, and the convergence rate $O\left(N^{-1 / 2}\right)$ can be obtained. Obviously, the error estimators and the errors derived in this paper are smaller than that achieved by [2]. Therefore, from the perspective of error, the HDG result of this paper is better than the FEM result of [2] for the suitable choice of $\tau_{0}$. 

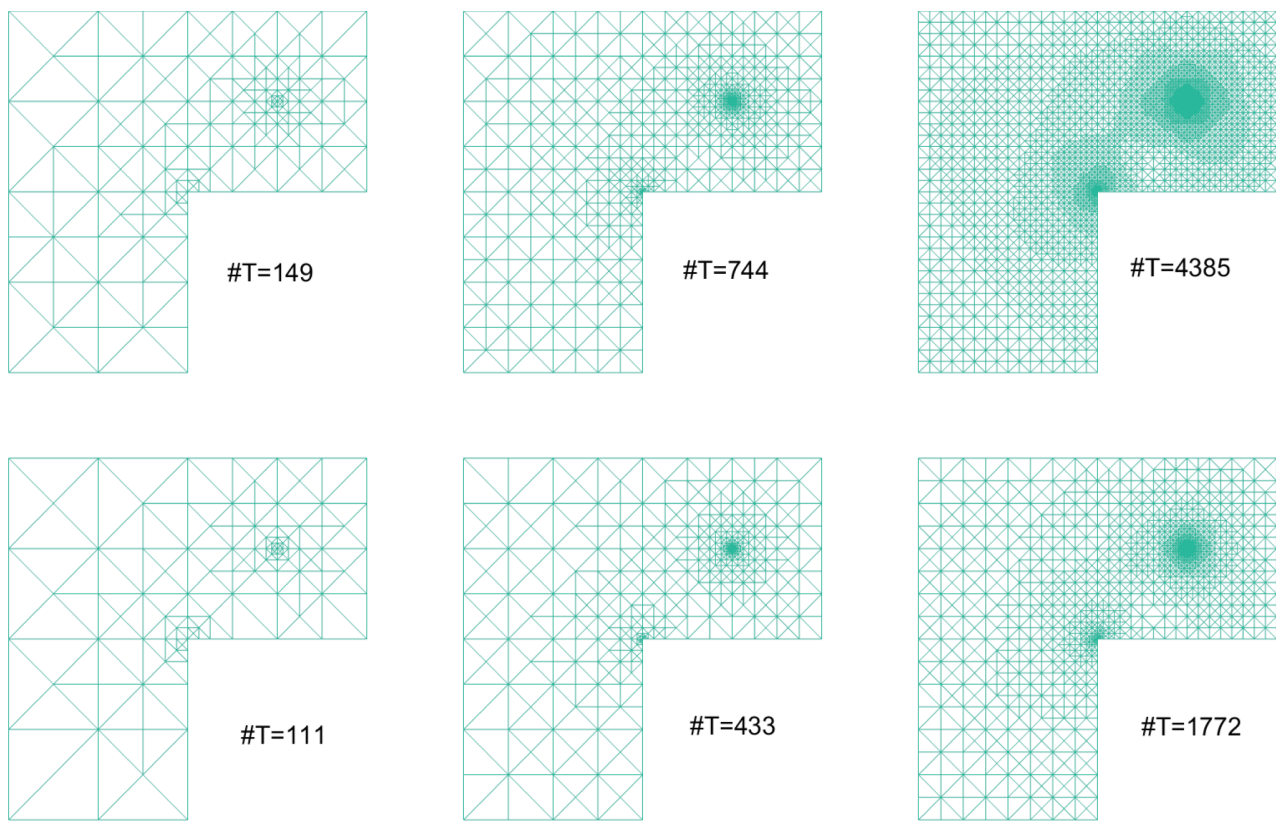

Figure 8 . Top: The adaptive meshes, generated by $\zeta_{1.3}$, after 13,19 and 25 iterations. Bottom: The adaptive meshes, generated by $\zeta_{1.5}$, after 13,19 and 25 iterations. Here, we set $\gamma=0.3$ and $k=1$.
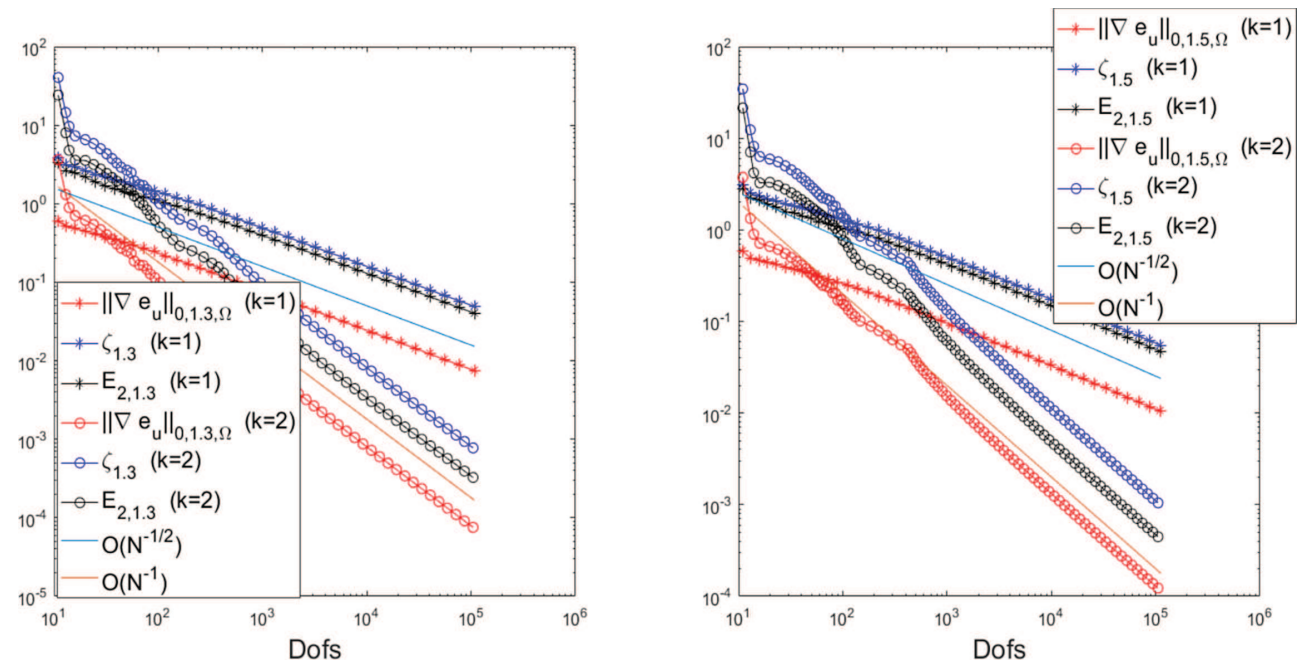

Figure 9. Left: The convergence histories of $\left\|\nabla e_{u}\right\|_{0,1.3, \Omega}, \zeta_{1.3}$ and $E_{2,1.3}$ for $k=1$ and $k=2$. Right: The convergence histories of $\left\|\nabla e_{u}\right\|_{0,1.5, \Omega}, \eta_{1.5}$ and $E_{2,1.5}$ for $k=1$ and $k=2$. Here we set $\gamma=0.3$. 

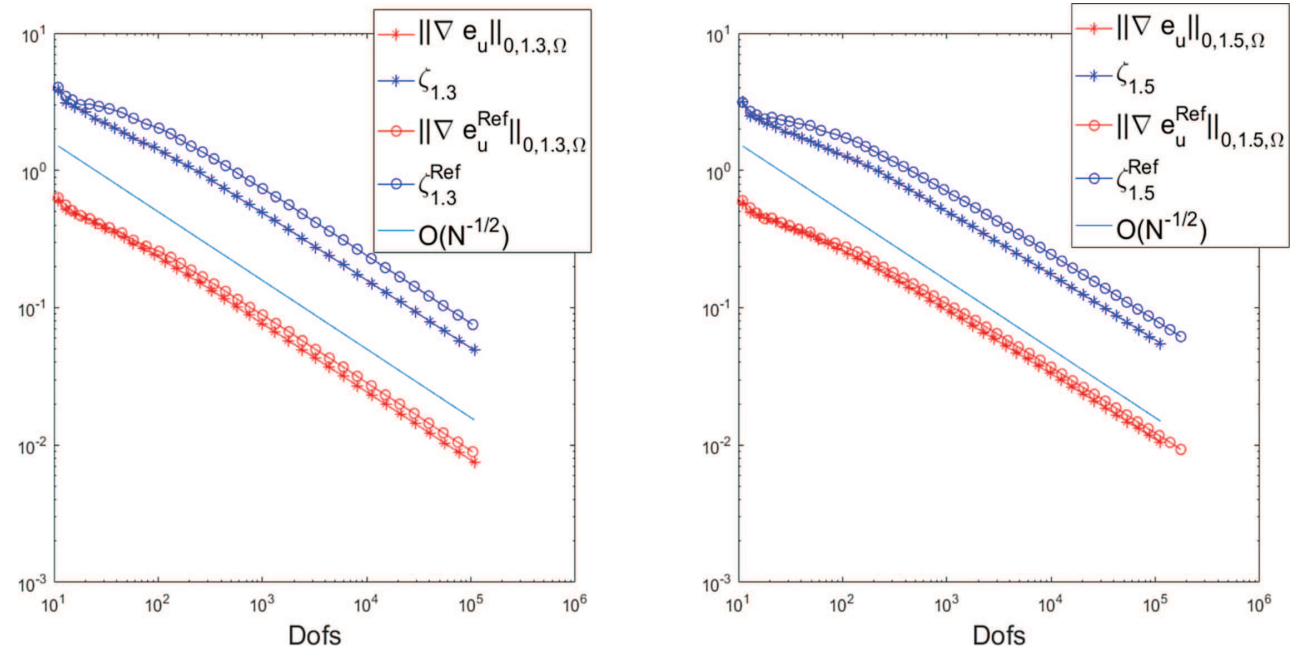

FiguRE 10. The convergence histories for $s=1.3$ and $s=1.5$.

\section{Conclusions}

In this paper, we investigate HDG methods for elliptic problems with Dirac measures. Firstly, a priori error estimate with convergence rate $O(h)$ is proved for the error in $L^{2}$-norm. Then, by duality argument and Oswald interpolation, the efficient and reliable a posteriori error estimators for the errors in $L^{2}$-norm and $W^{1, p}$-seminorm are obtained.

Finally the obtained a posteriori error estimators are used to design adaptive HDG algorithm, and some numerical examples are provided to verify the theoretical analysis and show the performance of the obtained a posteriori error estimators. By the numerical results, we find that the HDG scheme and error estimators of this paper are slightly better than the finite element discretization and error estimators of [2] based on a suitable choice of $\tau_{0}$, see Figures 2 and 10.

Acknowledgements. The work was supported by the NSF of China (Grant No. 12001209, 41974133) and the State Key Program of NSF of China (Grant No. 11931003).

\section{REFERENCES}

[1] R.A. Adams, Sobolev spaces. Academic Press (1975).

[2] R. Araya, E. Behrens and R. Rodríguez, A posteriori error estimates for elliptic problems with Dirac delta source terms. Numer. Math. 105 (2006) 193-216.

[3] R. Araya, E. Behrens and R. Rodríguez, An adaptive stabilized finite element scheme for a water quality model. Comput. Methods Appl. Mech. Engry. 196 (2007) 2800-2812.

[4] R. Araya, M. Solano and P. Vega, A posteriori error analysis of an HDG method for the Oseen problem. Appl. Numer. Math. 146 (2019) 291-308.

[5] R. Araya, M. Solano and P. Vega, Analysis of an adaptive HDG method for the Brinkman problem. IMA J. Numer. Anal. 39 (2019) 1502-1528.

[6] T. Apel, O. Benedix, D. Sirch and B. Vexler, A priori mesh grading for an elliptic problem with Dirac right-hand side. SIAM J. Numer. Anal. 49 (2011) 992-1005.

[7] J.P. Agnelli, E.M. Garau and P. Morin, A posteriori error estimates for elliptic problems with Dirac measure terms in weighted spaces. ESAIM: M2AN 48 (2014) 1557-1581.

[8] I. Babuška, Error bounds for the finite element method. Numer. Math. 16 (1971) 322-333.

[9] S.C. Brenner and L.R. Scott, The mathematical theory of finite element methods. Springer-Verlag, New York (2008).

[10] P.G. Ciarlet, The finite element methods for elliptic problems. Stud. Math. Appl. vol. 4, North-Holland, Amsterdam (1978). 
[11] E. Casas, $L^{2}$ estimates for the finite element method for the Dirchlet problem with singular data. Numer. Math. 47 (1985) $627-632$.

[12] E. Casas, Control of an elliptic problem with pointwise state constraints. SIAM J. Control Optim. 24 (1986) $1309-1318$.

[13] B. Cockburn and W. Zhang, A posteriori error estimates for HDG methods. J. Sci. Comput. 51 (2012) 582-607.

[14] B. Cockburn and W. Zhang, A posteriori error analysis for hybridizable discontinuous Galerkin methods for second order elliptic problems. SIAM J. Numer. Anal. 51 (2013) 676-693.

[15] B. Cockburn, J. Gopalakrishnan and R. Lazarov, Unified hybridization of discontinuous Galerkin, mixed, and continuous Galerkin methods for second order elliptic problems. SIAM J. Numer. Anal. 47 (2009) 1319-1365.

[16] B. Cockburn, J. Gopalakrishnan, N.C. Nguyen, J. Peraire and F.-J. Sayas, Analysis of HDG methods for Stokes flow. Math. Comput. 80 (2011) 723-760.

[17] H. Chen, J. Li and W. Qiu, A posteriori error estimates for HDG method for convection-diffusion equations. IMA J. Numer. Anal. 36 (2016) 437-462.

[18] G. Chen, W. Hu, J. Shen, J.R. Singler, Y. Zhang and X. Zheng, An HDG method for distributed control of convection diffusion PDEs. J. Comput. Appl. Math. 343 (2018) 643-661.

[19] K. Eriksson, Improve accuarcy by adapted mesh-refinements in the finite element method. Math. Comput. 44 (1985) 321-343.

[20] F. Fuica, F. Lepe, E. Otárola and D. Quero, A posteriori error estimates in $W^{1, p} \times L^{p}$ spaces for the Stokes system with Dirac measures. Preprint arXiv: 1912.08325 (2019).

[21] G. Fu, W. Qiu and W. Zhang, An analysis of HDG methods for convection-dominated diffusion problems. ESAIM: M2AN 49 (2015) 225-256.

[22] W. Gong, G. Wang and N. Yan, Approximation of elliptic optimal control problems acting on a lower dimensional manifold. SIAM J. Control Optim. 52 (2014) 2008-2035.

[23] W. Gong, W. Hu, M. Mateos, J.R. Singler, X. Zheng and Y. Zhang, A new HDG method for Dirichlet boundary control of convection diffusion PDEs II: low regularity. SIAM J. Numer. Anal. 56 (2018) 2262-2287.

[24] P. Grisvard, Elliptic problems for non-smooth domains. Pitman, Boston (1985).

[25] F.D. Gaspoz, P. Morin and A. Veeser, A posteriori error estimates with point sources in fractional Sobolev spaces. Numer. Methods Part. Diff. Equ. 33 (2017) 1018-1042.

[26] P. Houston and T.P. Wihler, Discontinuous Galerkin methods for problems with Dirac delta source. ESAIM: M2AN 46 (2012) $1467-1483$.

[27] O.A. Karakashian and F. Pascal, A posteriori error estimates for a discontinuous Galerkin approximation of second-order elliptic problems. SIAM J. Numer. Anal. 41 (2003) 2374-2399.

[28] H. Leng, Adaptive HDG methods for the steady-state incompressible Navier-Stokes equations. J. Sci. Comput. 87 (2021) 37.

[29] H. Leng and H. Chen, Adaptive HDG methods for the Brinkman equations with application to optimal control. J. Sci. Comput. 87 (2021) 46.

[30] I. Oikawa, HDG methods for second order elliptic problems. RIMS Kokyuroku 2037 (2017) 61-74.

[31] M. Petzoldt, A posteriori error estimators for elliptic equations with discontinuous coefficients. Adv. Comput. Math. 16 (2002) $47-75$.

[32] W. Qiu and K. Shi, A superconvergent HDG method for the incompressible Navier-Stokes equations on general polyhedral meshes. IMA J. Numer. Anal. 36 (2016) 1943-1967.

[33] R. Scott, Finite element convergence for singular data. Numer. Math. 21 (1973) 317-327.

[34] A.H. Schatz and L.B. Wahlbin, Interior maxumum norm estimates for finite element methods. Math. Comput. 31 (1977) $414-442$.

[35] R. Verfürth, A posteriori error estimators for convection-diffusion equations. Numer. Math. 80 (1998) $641-663$.

\section{Subscribe to Open (S2O)}

\section{A fair and sustainable open access model}

This journal is currently published in open access under a Subscribe-to-Open model (S2O). S2O is a transformative model that aims to move subscription journals to open access. Open access is the free, immediate, online availability of research articles combined with the rights to use these articles fully in the digital environment. We are thankful to our subscribers and sponsors for making it possible to publish this journal in open access, free of charge for authors.

\section{Please help to maintain this journal in open access!}

Check that your library subscribes to the journal, or make a personal donation to the $\mathrm{S} 2 \mathrm{O}$ programme, by contacting subscribers@edpsciences.org

More information, including a list of sponsors and a financial transparency report, available at: https://www. edpsciences.org/en/maths-s2o-programme 